\title{
MAPPING CLASS GROUP DYNAMICS ON SURFACE GROUP REPRESENTATIONS
}

\author{
WILLIAM M. GOLDMAN
}

\begin{abstract}
Deformation spaces $\operatorname{Hom}(\pi, G) / G$ of representations of the fundamental group $\pi$ of a surface $\Sigma$ in a Lie group $G$ admit natural actions of the mapping class group $\operatorname{Mod}_{\Sigma}$, preserving a Poisson structure. When $G$ is compact, the actions are ergodic. In contrast if $G$ is noncompact semisimple, the associated deformation space contains open subsets containing the Fricke-Teichmüller space upon which $\operatorname{Mod}_{\Sigma}$ acts properly. Properness of the $\operatorname{Mod}_{\Sigma^{-}}$ action relates to (possibly singular) locally homogeneous geometric structures on $\Sigma$. We summarize known results and state open questions about these actions.
\end{abstract}

\section{Contents}

Introduction $\quad 2$

Acknowledgments 3

1. Generalities 4

1.1. The Symplectic Structure 4

1.2. The Complex Case 5

1.3. Singularities of the deformation space 6

1.4. Surfaces with boundary 9

1.5. Examples of relative $\mathrm{SL}(2, \mathbb{C})$-character varieties 10

2. Compact Groups 13

2.1. Ergodicity 13

2.2. The unitary representation 14

2.3. Holomorphic objects 15

2.4. Automorphisms of free groups 16

2.5. Topological dynamics 16

Date: January 28, 2006.

1991 Mathematics Subject Classification. Primary: 57M50; Secondary: 58E20, $53 \mathrm{C} 24$.

Key words and phrases. Mapping class group, Riemann surface, fundamental group, representation variety, harmonic map, Teichmüller space, quasi-Fuchsian group, real projective structure, moduli space of vector bundles, hyperbolic manifold.

Goldman supported in part by NSF grants DMS-0103889 and DMS-0405605. 
2.6. Individual elements 17

3. Noncompact Groups and Uniformizations 17

3.1. Fricke-Teichmüller space 18

3.2. Other components and the Euler class 19

$\begin{array}{ll}\text { 3.3. The one-holed torus } & 19\end{array}$

3.4. Hyperbolic 3-manifolds 22

3.5. Convex Projective Structures and Hitchin representations 24

3.6. The energy of harmonic maps 24

3.7. Singular uniformizations and complex projective structures 25

$\begin{array}{ll}\text { 3.8. Complex projective structures } & 27\end{array}$

References 28

\section{INTRODUCTION}

A natural object associated to a topological surface $\Sigma$ is the deformation space of representations of its fundamental group $\pi=\pi_{1}(\Sigma)$ in a Lie group $G$. These spaces admit natural actions of the mapping class group $\operatorname{Mod}_{\Sigma}$ of $\Sigma$, and therefore determine linear representations of $\operatorname{Mod}_{\Sigma}$.

The purpose of this paper is to survey recent results on the dynamics of these actions, and speculate on future directions in this subject.

The prototypes of this theory are two of the most basic spaces in Riemann surface theory: the Jacobian and the Fricke-Teichmüller space. The Jacobian $\operatorname{Jac}(M)$ of a Riemann surface $M$ homeomorphic to $\Sigma$ identifies with the deformation space $\operatorname{Hom}(\pi, G) / G$ when $G$ is the circle U(1). The Jacobian parametrizes topologically trivial holomorphic complex line bundles over $M$, but its topological type (and symplectic structure) are invariants of the underlying topological surface $\Sigma$. The action of $\operatorname{Mod}_{\Sigma}$ is the action of the integral symplectic group $\operatorname{Sp}(2 g, \mathbb{Z})$ on the torus $\mathbb{R}^{2 g} / \mathbb{Z}^{2 g}$, which is a measure-preserving chaotic (ergodic) action.

In contrast, the Teichmüller space $\mathfrak{T}_{\Sigma}$ (Fricke space if $\left.\partial \Sigma \neq \emptyset\right)$ is comprised of equivalence classes of marked conformal structures on $\Sigma$. A marked conformal structure is a pair $(M, f)$ where $f$ is a homeomorphism and $M$ is a Riemann surface. Marked conformal structures $\left(f_{1}, M_{1}\right)$ and $\left(f_{2}, M_{2}\right)$ are equivalent if there is a biholomorphism $M_{1} \stackrel{h}{\rightarrow} M_{2}$ such that $h \circ f_{1}$ is homotopic to $f_{2}$. Denote the equivalence class of a marked conformal structure $(f, M)$ by

$$
\langle f, M\rangle \in \mathfrak{T}_{\Sigma}
$$


A marking $f$ determines a representation of the fundamental group:

$$
\pi=\pi_{1}(\Sigma) \stackrel{f_{*}}{\longrightarrow} \pi_{1}(M) \subset \operatorname{Aut}(\tilde{M}) .
$$

By the uniformization theorem (at least when $\chi(\Sigma)<0$ ), these identify with marked hyperbolic structures on $\Sigma$, which in turn identify with conjugacy classes of discrete embeddings of the fundamental group $\pi$ in the group $G=\operatorname{PGL}(2, \mathbb{R})$ of isometries of the hyperbolic plane. These classes form a connected component of $\operatorname{Hom}(\pi, G) / G$, which is homeomorphic to a cell of dimension $-3 \chi(\Sigma)$ [35]. The mapping class group $\operatorname{Mod}_{\Sigma}$ acts properly on $\mathfrak{T}_{\Sigma}$. The quotient orbifold

$$
\mathfrak{M}_{\Sigma}:=\mathfrak{T}_{\Sigma} / \operatorname{Mod}_{\Sigma}
$$

is the Riemann moduli space, consisting of biholomorphism classes of (unmarked) conformal structures on $\Sigma$.

Summarizing:

- When $G$ is compact, $\operatorname{Hom}(\pi, G) / G$ has nontrivial homotopy type, and the action of the mapping class group exhibits nontrivial dynamics;

- When $G=\operatorname{PGL}(2, \mathbb{R})$ (or more generally a noncompact semisimple Lie group), $\operatorname{Hom}(\pi, G) / G$ contains open sets (like Teichmüller space) which are contractible and admit a proper $\operatorname{Mod}_{\Sigma}$-action. Often these open sets correspond to locally homogeneous geometric structures uniformizing $\Sigma$.

Thus dynamically complicated mapping class group actions accompany nontrivial homotopy type of the deformation space. In general the dynamics exhibits properties of these two extreme cases, as will be described in this paper.

Acknowledgments. This paper is an expanded version of a lecture presented at the Special Session "Dynamics of Mapping Class Group Actions" at the Annual Meeting of the American Mathematical Society, January 6-11, 2005, in Atlanta, Georgia. I am grateful to Richard Brown for organizing this workshop, and the opportunity to lecture on this subject. I am also grateful to Benson Farb for encouraging me to write this paper, and to Jørgen Andersen, David Dumas, Lisa Jeffrey, Misha Kapovich, François Labourie, Dan Margalit, Howard Masur, Walter Neumann, Juan Souto, Pete Storm, Ser Tan, Richard Wentworth, Anna Wienhard and Eugene Xia for several suggestions and helpful comments. I wish to thank the referee for a careful reading of the paper and many useful suggestions. 


\section{Generalities}

Let $\pi$ be a finitely generated group and $G$ a real algebraic Lie group. The set $\operatorname{Hom}(\pi, G)$ of homomorphisms $\pi \longrightarrow G$ has the natural structure of an affine algebraic set. The group

$$
\operatorname{Aut}(\pi) \times \operatorname{Aut}(G)
$$

acts on $\operatorname{Hom}(\pi, G)$ by left- and right- composition, preserving the algebraic structure: if $\alpha \in \operatorname{Aut}(\pi)$ and $h \in \operatorname{Aut}(G)$ are automorphisms, then the action of $(\alpha, h)$ on $\rho \in \operatorname{Hom}(\pi, G)$ is the composition $h \circ \rho \circ \alpha^{-1}$ :

$$
\pi \stackrel{\alpha^{-1}}{\longrightarrow} \pi \stackrel{\rho}{\rightarrow} G \stackrel{h}{\rightarrow} G
$$

The deformation space is the quotient space of $\operatorname{Hom}(\pi, G)$ (with the classical topology) by the subgroup $\operatorname{lnn}(G)$ of inner automorphisms of $G$, and is denoted $\operatorname{Hom}(\pi, G) / G$. The action of the inner automorphism $\iota_{\gamma}$ determined by an element $\gamma \in \pi$ equals $\iota_{\rho\left(\gamma^{-1}\right)}(\rho)$. Therefore $\operatorname{lnn}(\pi)$ acts trivially on $\operatorname{Hom}(\pi, G) / G$ and the induced action of $\operatorname{Aut}(\pi)$ on $\operatorname{Hom}(\pi, G) / G$ factors through the quotient

$$
\operatorname{Out}(\pi):=\operatorname{Aut}(\pi) / \operatorname{lnn}(\pi) \text {. }
$$

When $\Sigma$ is a closed orientable surface with $\chi(\Sigma)<0$, then the natural homomorphism

$$
\pi_{0}(\operatorname{Diff}(\Sigma)) \longrightarrow \operatorname{Out}(\pi)
$$

is an isomorphism. The mapping class group $\operatorname{Mod}_{\Sigma}$ is the subgroup of Out $(\pi)$ corresponding to orientation-preserving diffeomorphisms of $\Sigma$.

When $\Sigma$ has nonempty boundary with components $\partial_{i} \Sigma$, this deformation space admits a boundary restriction map

$$
\left.\operatorname{Hom}\left(\pi_{1}(\Sigma), G\right) / G \longrightarrow \prod_{i \in \pi_{0}(\partial \Sigma)} \operatorname{Hom}\left(\pi_{1}\left(\partial_{i} \Sigma\right)\right), G\right) / G
$$

The fibers of the boundary restriction map are the relative character varieties. This action of $\operatorname{Mod}_{\Sigma}$ preserves this map.

1.1. The Symplectic Structure. These spaces possess algebraic symplectic structures, invariant under $\operatorname{Mod}_{\Sigma}$. For the moment we focus on the smooth part of $\operatorname{Hom}(\pi, G)$, which we define as follows. When $G$ is reductive, the subset $\operatorname{Hom}(\pi, G)^{--}$consisting of representations whose image does not lie in a parabolic subgroup of $G$ is a smooth submanifold upon which $\operatorname{lnn}(G)$ acts properly and freely. The quotient $\operatorname{Hom}(\pi, G)^{--} / G$ is then a smooth manifold, with a $\operatorname{Mod}_{\Sigma}$-invariant symplectic structure. 
The symplectic structure depends on a choice of a nondegenerate Ad-invariant symmetric bilinear form $\mathbb{B}$ on the Lie algebra $\mathfrak{g}$ of $G$ and an orientation on $\Sigma$. The composition

$$
\pi \stackrel{\rho}{\longrightarrow} G \stackrel{\text { Ad }}{\longrightarrow} \operatorname{Aut}(\mathfrak{g})
$$

defines a $\pi$-module $\mathfrak{g}_{\text {Ad } \rho}$. The Zariski tangent space to $\operatorname{Hom}(\pi, G)$ at a representation $\rho$ is the space $Z^{1}\left(\pi, \mathfrak{g}_{\mathrm{Ad} \rho}\right)$ of 1 -cocycles. The tangent space to the orbit $G \rho$ equals the subspace $B^{1}\left(\pi, \mathfrak{g}_{\mathrm{Ad} \rho}\right)$ of 1 -coboundaries. These facts are due to Weil [102], see also Raghunathan [88]. If $G$ acts properly and freely on a neighborhood of $\rho$ in $\operatorname{Hom}(\pi, G)$, then $\operatorname{Hom}(\pi, G) / G$ is a manifold near $[\rho]$ with tangent space $H^{1}\left(\pi, \mathfrak{g}_{\text {Ad } \rho}\right)$. In that case a nondegenerate symmetric $\operatorname{Ad}(G)$-invariant bilinear form

$$
\mathfrak{g} \times \mathfrak{g} \stackrel{\mathbb{B}}{\rightarrow} \mathbb{R}
$$

defines a pairing of $\pi$-modules

$$
\mathfrak{g}_{\text {Ad } \rho} \times \mathfrak{g}_{\text {Ad } \rho} \stackrel{\mathbb{B}}{\rightarrow} \mathbb{R} .
$$

Cup product using $\mathbb{B}$ as coefficient pairing defines a nondegenerate skew-symmetric pairing

$$
H^{1}\left(\pi, \mathfrak{g}_{\mathrm{Ad} \rho}\right) \times H^{1}\left(\pi, \mathfrak{g}_{\mathrm{Ad} \rho}\right) \stackrel{\mathbb{B}_{*}(\cup)}{\longrightarrow} H^{2}(\pi, \mathbb{R}) \cong \mathbb{R}
$$

on each tangent space

$$
T_{[\rho]} \operatorname{Hom}(\pi, G) / G \cong H^{1}\left(\pi, \mathfrak{g}_{\mathrm{Ad} \rho}\right) .
$$

Here the isomorphism $H^{2}(\pi, \mathbb{R}) \cong \mathbb{R}$ arises from the orientation on $\Sigma$. The resulting exterior 2 -form $\omega_{\mathbb{B}}$ is closed [36], and defines a symplectic structure on the smooth part $\operatorname{Hom}(\pi, G)^{--} / G$ of $\operatorname{Hom}(\pi, G) / G$. This topological definition makes it apparent that $\omega_{\mathbb{B}}$ is $\operatorname{Mod}_{\Sigma}$-invariant. In particular the action preserves the measure $\mu$ defined by $\omega_{\mathbb{B}}$. When $G$ is compact, the total measure is finite (Jeffrey-Weitsman [61, 62], Huebschmann [60]).

1.2. The Complex Case. When $G$ is a complex Lie group, $\operatorname{Hom}(\pi, G)$ has a complex algebraic structure preserved by the $\operatorname{Aut}(\pi) \times \operatorname{Aut}(G)$ action. When $G$ is a complex semisimple Lie group, the above construction, applied to a nondegenerate Ad-invariant complex-bilinear form

$$
\mathfrak{g} \times \mathfrak{g} \stackrel{\mathbb{B}}{\rightarrow} \mathbb{C},
$$

determines a complex-symplectic structure on $\operatorname{Hom}(\pi, G)^{--} / G$, that is, a closed nondegenerate holomorphic $(2,0)$-form. This complexsymplectic structure is evidently Mod $_{\Sigma}$-invariant. For a discussion of this structure when $G=\operatorname{SL}(2, \mathbb{C})$, see [45]. 
The choice of a marked conformal structure on $\Sigma$ determines a hyperKähler structure on $\operatorname{Hom}(\pi, G) / G$ subordinate to this complex-symplectic structure.

A complex-symplectic structure on a $4 m$-dimensional real manifold $V$ is given by an integrable almost complex structure $J$ and a closed nondegenerate skew-symmetric bilinear form

$$
T M \times T M \stackrel{\Omega}{\rightarrow} \mathbb{C}
$$

which is complex-bilinear with respect to $J$. Alternatively, it is defined by a reduction of the structure group of the tangent bundle $T V$ from $\mathrm{GL}(4 m, \mathbb{R})$ to the subgroup

$$
\operatorname{Sp}(2 m, \mathbb{C}) \subset \mathrm{GL}(4 m, \mathbb{R}) .
$$

A hyper-Kähler structure further reduces the structure group of the tangent bundle from $\operatorname{Sp}(2 m, \mathbb{C})$ to its maximal compact subgroup $\mathrm{Sp}(2 m) \subset \mathrm{Sp}(2 m, \mathbb{C})$. All of these structures are required to satisfy certain integrability conditions. A hyper-Kähler structure subordinate to a complex-symplectic structure $(\Omega, J)$ is defined by a Riemannian metric $g$ and integrable almost complex structures $I, K$ such that:

- $g$ is Kählerian with respect to each of $I, J, K$,

- the complex structures $I, J, K$ satisfy the quaternion identities,

- $\Omega(X, Y)=-g(I X, Y)+i g(K X)$ for $X, Y \in T M$.

Goldman-Xia [54], $§ 5$ describes this structure in detail when $G=$ $\mathrm{GL}(1, \mathbb{C})$.

From this we can associate to every point in Teichmüller space $\mathfrak{T}_{\Sigma}$ a compatible hyper-Kähler structure on the complex-symplectic space $\operatorname{Hom}(\pi, G)^{--} / G$. However the hyper-Kähler structures are not $\operatorname{Mod}_{\Sigma^{-}}$ invariant.

1.3. Singularities of the deformation space. In general the spaces $\operatorname{Hom}(\pi, G)$ and $\operatorname{Hom}(\pi, G) / G$ are not manifolds, but their local structure admits a very explicit cohomological description. For convenience assume that $G$ is reductive algebraic and that $\rho$ is a reductive representation, that is, its image $\rho(\pi)$ is Zariski dense in a reductive subgroup of $G$. For $\rho \in \operatorname{Hom}(\pi, G)$, denote the centralizer of $\rho(\pi)$ by $\mathfrak{Z}(\rho)$ and the center of $G$ by $\mathfrak{Z}$.

A representation $\rho \in \operatorname{Hom}(\pi, G)$ is a singular point of $\operatorname{Hom}(\pi, G)$ if and only if

$$
\operatorname{dim}(\mathfrak{Z}(\rho) / \mathfrak{Z})>0
$$

Equivalently, the isotropy group of $\operatorname{lnn}(G)$ at $\rho$ is not discrete, that is, the action of $\operatorname{lnn}(G)$ at $\rho$ is not locally free. 
The Zariski tangent space $T_{\rho} \operatorname{Hom}(\pi, G)$ equals the space $Z^{1}\left(\pi ; \mathfrak{g}_{\text {Ad } \rho}\right)$

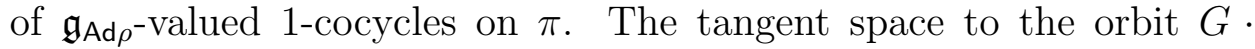
$\rho$ equals the subspace $B^{1}\left(\pi ; \mathfrak{g}_{\text {Ad } \rho}\right)$ of coboundaries. Thus the Zariski normal space at $\rho$ to the orbit $G \cdot \rho$ in $\operatorname{Hom}(\pi, G)$ equals the cohomology group $H^{1}\left(\pi ; \mathfrak{g}_{\text {Ad } \rho}\right)$.

Here is a heuristic interpretation. Consider an analytic path $\rho_{t} \in$ $\operatorname{Hom}(\pi, G)$ with $\rho_{0}=\rho$. Expand it as a power series in $t$ :

$$
\rho_{t}(x)=\exp \left(u_{0}(x) t+u_{2}(x) t^{2}+u_{3}(x) t^{3}+\ldots\right) \rho(x)
$$

where

$$
\pi \stackrel{u_{n}}{\longrightarrow} \mathfrak{g}
$$

for $n \geq 0$. The condition

$$
\rho_{t}(x y)=\rho_{t}(x) \rho_{t}(y)
$$

implies that the tangent vector $u=u_{0}$ satisfies the cocycle condition

$$
u(x y)=u(x)+\operatorname{Ad} \rho(x) u(y),
$$

(the linearization of (1.3). The vector space of solutions of (1.4) is the space $Z^{1}\left(\pi ; \mathfrak{g}_{\text {Ad } \rho}\right)$ of $\mathfrak{g}_{\text {Ad } \rho}$-valued 1-cocycles of $\pi$.

The Zariski tangent space to the orbit $G \cdot \rho$ equals the subspace $B^{1}\left(\pi, \mathfrak{g}_{\text {Ad } \rho}\right) \subset Z^{1}\left(\pi, \mathfrak{g}_{\text {Ad } \rho}\right)$ consisting of 1-coboundaries. Suppose that a path $\rho_{t}$ in $\operatorname{Hom}(\pi, G)$ is induced by a conjugation by a path $g_{t}$

$$
\rho_{t}(x)=g_{t} \rho(x) g_{t}^{-1}
$$

where $g_{t}$ admits a power series expansion

$$
g_{t}=\exp \left(v_{1} t+v_{2} t^{2}+\ldots\right),
$$

where $v_{1}, v_{2}, \cdots \in \mathfrak{g}$. Thus the tangent vector to $\rho_{t}$ is tangent to the orbit $G \cdot \rho$. Expanding the power series, this tangent vector equals

$$
u(x)=v_{1}-\operatorname{Ad} \rho(x) v_{1},
$$

that is, $u=\delta v_{1} \in B^{1}\left(\pi ; \mathfrak{g}_{\mathrm{Ad} \rho}\right)$ is a coboundary.

Let $u \in T_{\rho} \operatorname{Hom}(\pi, G)=Z^{1}\left(\pi ; \mathfrak{g}_{\text {Ad } \rho}\right)$ be a tangent vector to $\operatorname{Hom}(\pi, G)$ at $\rho$. We give necessary and sufficient conditions that $u$ be tangent to an analytic path of representations.

Solving the equation (1.3) to second order gives:

$$
u_{2}(x)-u_{2}(x y)+\operatorname{Ad} \rho(x) u_{2}(y)=\frac{1}{2}[u(x), \operatorname{Ad} \rho(x) u(y)] .
$$

Namely, the function,

$$
\begin{aligned}
\pi \times \pi & \mathfrak{g} \\
(x, y) & \longmapsto \frac{1}{2}[u(x), \operatorname{Ad} \rho(x) u(y)]
\end{aligned}
$$


is a $\mathfrak{g}_{\text {Ad } \rho}$-valued 2-cochain on $\pi$, This 2-cochain is the coboundary $\delta u_{2}$ of the 1-cochain $\pi \stackrel{u_{2}}{\longrightarrow} \mathfrak{g}_{\mathrm{Ad} \rho}$. Similarly there are conditions on the coboundary of $u_{n}$ in terms of the lower terms in the power series expansion (1.2).

The operation (1.6) has a cohomological interpretation as follows. $\pi$ acts on $\mathfrak{g}$ by Lie algebra automorphisms, so that Lie bracket defines a pairing of $\pi$-modules

$$
\mathfrak{g}_{\mathrm{Ad} \rho} \times \mathfrak{g}_{\mathrm{Ad} \rho} \stackrel{[,]}{\rightarrow} \mathfrak{g}_{\mathrm{Ad} \rho} .
$$

The Lie algebra of $\mathfrak{Z}(\rho)$ equals $H^{0}\left(\pi ; \mathfrak{g}_{\mathrm{Ad} \rho}\right)$. The linearization of the action of $\mathfrak{Z}(\rho)$ is given by the cup product on $H^{1}\left(\pi ; \mathfrak{g}_{\text {Ad } \rho}\right)$ with [,] as coefficient pairing:

$$
H^{0}\left(\pi ; \mathfrak{g}_{\mathrm{Ad} \rho}\right) \times H^{1}\left(\pi ; \mathfrak{g}_{\mathrm{Ad} \rho}\right) \stackrel{[,]_{*}(\cup)}{\longrightarrow} H^{1}\left(\pi ; \mathfrak{g}_{\mathrm{Ad} \rho}\right) .
$$

Now consider the cup product of 1-dimensional classes. The bilinear form

$$
H^{1}\left(\pi ; \mathfrak{g}_{\mathrm{Ad} \rho}\right) \times H^{1}\left(\pi ; \mathfrak{g}_{\mathrm{Ad} \rho}\right) \stackrel{[,]_{*}(\cup)}{\longrightarrow} H^{2}\left(\pi ; \mathfrak{g}_{\mathrm{Ad} \rho}\right) .
$$

is symmetric; let $Q_{\rho}$ be the associated quadratic form.

Suppose $u$ is tangent to an analytic path. Solving (1.2) to second order (as in (1.5) and (1.6)) implies that

$$
\left.[,]_{*}(\cup)([u],[u]]\right)=\delta u_{2},
$$

that is,

$$
Q_{\rho}([u])=0 .
$$

Under the above hypotheses, the necessary condition (1.7) is also sufficient. In fact, by Goldman-Millson [50], $\rho$ has a neighborhood $N$ in $\operatorname{Hom}(\pi, G)$ analytically equivalent to a neighborhood of 0 of the cone $C_{\rho}$ in $Z^{1}\left(\pi ; \mathfrak{g}_{\mathrm{Ad} \rho}\right)$ defined by the homogeneous quadratic function

$$
\begin{aligned}
Z^{1}\left(\pi ; \mathfrak{g}_{\mathrm{Ad} \rho}\right) & \longrightarrow H^{2}\left(\pi ; \mathfrak{g}_{\mathrm{Ad} \rho}\right) \\
u & \longmapsto Q_{\rho}([u]) .
\end{aligned}
$$

Then the germ of $\operatorname{Hom}(\pi, G) / G$ at $[\rho]$ is the quotient of this cone by the isotropy group $\mathfrak{Z}(\rho)$. (These spaces are special cases of symplectic stratified spaces of Sjamaar-Lerman [79].)

An explicit exponential mapping

$$
N \stackrel{\operatorname{Exp}_{\rho}}{\longrightarrow} \operatorname{Hom}(\pi, G)
$$

was constructed by Goldman-Millson [49] using the Green's operator of a Riemann surface $M$ homeomorphic to $\Sigma$.

The subtlety of these constructions is underscored by the following false argument, which seemingly proves that the Torelli subgroup of 
$\operatorname{Mod}_{\Sigma}$ acts identically on the whole component of $\operatorname{Hom}(\pi, G) / G$ containing the trivial representation. This is easily seen to be false, for $G$ semisimple.

Here is the fallacious argument. The trivial representation $\rho_{0}$ is fixed by all of $\operatorname{Mod}_{\Sigma}$. Thus $\operatorname{Mod}_{\Sigma}$ acts on the analytic germ of $\operatorname{Hom}(\pi, G) / G$ at $\rho_{0}$. At $\rho_{0}$, the coefficient module $\mathfrak{g}_{\mathrm{Ad} \rho}$ is trivial, and the tangent space corresponds to ordinary (untwisted) cohomology:

$$
T_{\rho_{0}} \operatorname{Hom}(\pi, G)=Z^{1}(\pi ; \mathfrak{g})=Z^{1}(\pi) \otimes \mathfrak{g} .
$$

The quadratic form is just the usual cup-product pairing, so any homologically trivial automorphism $\phi$ fixes the quadratic cone $N$ pointwise. By Goldman-Millson [50], the analytic germ of $\operatorname{Hom}(\pi, G)$ at $\rho_{0}$ is equivalent to the quadratic cone $N$. Therefore $[\phi]$ acts trivially on an open neighborhood of $\rho$ in $\operatorname{Hom}(\pi, G)$. By analytic continuation, $[\phi]$ acts trivially on the whole component of $\operatorname{Hom}(\pi, G)$ containing $\rho$.

The fallacy arises because the identification $\operatorname{Exp}_{\rho}$ of a neighborhood $N$ in the quadratic cone with the germ of $\operatorname{Hom}(\pi, G)$ at $\rho$ depends on a choice of Riemann surface $M$. Each point $\langle f, M\rangle \in \mathfrak{T}_{\Sigma}$ determines an exponential map $\operatorname{Exp}_{\rho,\langle f, M\rangle}$ from the germ of the quadratic cone to $\operatorname{Hom}(\pi, G)$, and these are not invariant under $\operatorname{Mod}_{\Sigma}$. In particular, no family of isomorphisms of the analytic germ of $\operatorname{Hom}(\pi, G)$ at $\rho_{0}$ with the quadratic cone $N$ is $\operatorname{Mod}_{\Sigma}$-invariant.

Problem 1.1. Investigate the dependence of $\operatorname{Exp}_{\rho,\langle f, M\rangle}$ on the marked Riemann surface $\langle f, M\rangle$.

1.4. Surfaces with boundary. When $\Sigma$ has nonempty boundary, an Ad-invariant inner product $\mathbb{B}$ on $\mathfrak{g}$ and an orientation on $\Sigma$ determines a Poisson structure (Fock-Rosly [32], Guruprasad-Huebschmann-JeffreyWeinstein [55]). The symplectic leaves of this Poisson structure are the level sets of the boundary restriction map (1.1).

For each component $\partial_{i} \Sigma$ of $\partial \Sigma$, fix a conjugacy class $C_{i} \subset G$. The subspace

$$
\operatorname{Hom}(\pi, G) / G_{\left(C_{1}, \ldots, C_{b}\right)} \subset \operatorname{Hom}(\pi, G) / G
$$

consisting of $[\rho]$ such that

$$
\rho\left(\partial_{i} \Sigma\right) \subset C_{i}
$$

has a symplectic structure. (To simplify the discussion we assume that it is a smooth submanifold.) De Rham cohomology with twisted coefficients in $\mathfrak{g}_{\mathrm{Ad} \rho}$ is naturally isomorphic with group cohomology of $\pi$. In terms of De Rham cohomology, the tangent space at $[\rho]$ to 
$\operatorname{Hom}(\pi, G) / G_{\left(C_{1}, \ldots, C_{b}\right)}$ identifies with

$$
\begin{aligned}
& \operatorname{Ker}\left(H^{1}\left(\Sigma ; \mathfrak{g}_{\mathrm{Ad} \rho}\right)\right.\left.\rightarrow H^{1}\left(\partial \Sigma ; \mathfrak{g}_{\mathrm{Ad} \rho}\right)\right) \\
& \cong \operatorname{Image}\left(H^{1}\left(\Sigma, \partial \Sigma ; \mathfrak{g}_{\mathrm{Ad} \rho}\right) \rightarrow H^{1}\left(\Sigma ; \mathfrak{g}_{\mathrm{Ad} \rho}\right)\right) .
\end{aligned}
$$

The cup product pairing

$$
H^{1}\left(\Sigma ; \mathfrak{g}_{\mathrm{Ad} \rho}\right) \times H^{1}\left(\Sigma, \partial \Sigma ; \mathfrak{g}_{\mathrm{Ad} \rho}\right) \stackrel{\mathbb{B}_{*}(\cup)}{\longrightarrow} H^{2}(\Sigma, \partial \Sigma ; \mathbb{R})
$$

induces a symplectic structure on $\operatorname{Hom}(\pi, G) / G_{\left(C_{1}, \ldots, C_{b}\right)}$.

Given a (possibly singular) foliation $\mathfrak{F}$ of a manifold $X$ by symplectic manifolds, the Poisson structure is defined as follows. For functions $f, g \in C^{\infty}(X)$, their Poisson bracket is a function $\{f, g\}$ on $X$ defined as follows. Let $x \in X$ and let $L_{x}$ be the leaf of $\mathfrak{F}$ containing $x$. Define the value of $\{f, g\}$ at $x$ as the Poisson bracket

$$
\left\{\left.f\right|_{L_{x}},\left.g\right|_{L_{x}}\right\}_{L_{x}},
$$

where $\{,\}_{L_{x}}$ denotes the Poisson bracket operation on the symplectic manifold $L_{x}$, and $\left.f\right|_{L_{x}},\left.g\right|_{L_{x}} \in C^{\infty}\left(L_{x}\right)$, are the restrictions of $f, g$ to $L_{x}$.

The examples below exhibit exterior bivector fields $\xi$ representing the Poisson structure. If $f, g \in C^{\infty}(X)$, their Poisson bracket $\{f, g\}$ is expressed as an interior product of $\xi$ with the exterior derivatives of $f, g$ :

$$
\{f, g\}=\xi \cdot(d f \otimes d g) .
$$

In local coordinates $\left(x^{1}, \ldots, x^{n}\right)$, write

$$
\xi=\sum_{i, j} \xi^{i, j} \frac{\partial}{\partial x_{i}} \wedge \frac{\partial}{\partial x_{j}}
$$

with $\xi^{i, j}=-\xi^{j, i}$. Then

$$
\begin{aligned}
\{f, g\} & =\sum_{i, j}\left(\xi^{i, j} \frac{\partial}{\partial x_{i}} \wedge \frac{\partial}{\partial x_{j}}\right) \cdot\left(\frac{\partial f}{\partial x_{i}} d x^{i} \otimes \frac{\partial g}{\partial x_{j}} d x^{j}\right) \\
& =\sum_{i, j} \xi^{i, j}\left(\frac{\partial f}{\partial x_{i}} \frac{\partial g}{\partial x_{j}}-\frac{\partial f}{\partial x_{j}} \frac{\partial g}{\partial x_{i}}\right)
\end{aligned}
$$

1.5. Examples of relative $\operatorname{SL}(2, \mathbb{C})$-character varieties. We give a few explicit examples, when $G=\operatorname{SL}(2, \mathbb{C})$, and $\Sigma$ is a three-holed or four-holed sphere, or a one-holed or two-holed torus. Since generic conjugacy classes in $\mathrm{SL}(2, \mathbb{C})$ are determined by the trace function

$$
\operatorname{SL}(2, \mathbb{C}) \stackrel{\operatorname{tr}}{\rightarrow} \mathbb{C}
$$


the relative character varieties are level sets of the mapping

$$
\begin{aligned}
\operatorname{Hom}(\pi, G) / G & \longrightarrow \mathbb{C}^{b} \\
{[\rho] \longmapsto } & {\left[\operatorname{tr}\left(\rho\left(\partial_{i}(\Sigma)\right)\right]_{i=1, \ldots, b}\right.}
\end{aligned}
$$

1.5.1. The three-holed sphere. When $\Sigma$ is a three-holed sphere, its fundamental group admits a presentation

$$
\pi=\langle A, B, C \mid A B C=1\rangle
$$

where $A, B, C$ correspond to the three components of $\partial \Sigma$. Here is the fundamental result for $\operatorname{SL}(2, \mathbb{C})$-character varieties of a rank two free group:

Theorem (Vogt [101]-Fricke [33]). The map

$$
\begin{aligned}
\operatorname{Hom}(\pi, G) / G & \longrightarrow \mathbb{C}^{3} \\
{[\rho] \longmapsto } & {\left[\begin{array}{l}
\operatorname{tr}(\rho(A)) \\
\operatorname{tr}(\rho(B)) \\
\operatorname{tr}(\rho(C))
\end{array}\right] }
\end{aligned}
$$

is an isomorphism of affine varieties.

In particular, the symplectic leaves are just points. See [46] for an elementary proof..

1.5.2. The one-holed torus. When $\Sigma$ is a one-holed torus, its fundamental group admits a presentation

$$
\pi=\left\langle X, Y, Z, K \mid X Y Z=1, K=X Y X^{-1} Y^{-1}\right\rangle
$$

where $X, Y$ are simple loops intersecting once, and $K=X Y X^{-1} Y^{-1}$ corresponds to the boundary. Presenting the interior of $\Sigma$ as the quotient

$$
\operatorname{int}(\Sigma)=\left(\mathbb{R}^{2}-\mathbb{Z}^{2}\right) / \mathbb{Z}^{2}
$$

the curves $X, Y$ correspond to the $(1,0)$ and $(0,1)$-curves respectively. Once again the Vogt-Fricke theorem implies that $\operatorname{Hom}(\pi, G) / G \cong \mathbb{C}^{3}$, with coordinates

$$
\begin{aligned}
& x=\operatorname{tr}(\rho(X)) \\
& y=\operatorname{tr}(\rho(Y)) \\
& z=\operatorname{tr}(\rho(Z)) .
\end{aligned}
$$

The boundary trace $\operatorname{tr}(\rho(K))$ is:

$$
\kappa(x, y, z)=x^{2}+y^{2}+z^{2}-x y z-2
$$


so the relative character varieties are the level sets $\kappa^{-1}(t)$. This mapping class group $\operatorname{Mod}_{\Sigma}$ acts by polynomial transformations of $\mathbb{C}^{3}$, preserving the function $\kappa$ (compare [44]). The Poisson structure is given by the bivector field

$$
\begin{aligned}
d \kappa \cdot\left(\partial_{x} \wedge \partial_{y} \wedge \partial_{z}\right) & =(2 x-y z) \partial_{y} \wedge \partial_{z} \\
& +(2 y-z x) \partial_{z} \wedge \partial_{x} \\
& +(2 z-x y) \partial_{x} \wedge \partial_{y}
\end{aligned}
$$

(where $\partial_{x}$ denotes $\frac{\partial}{\partial x}$, etc.).

1.5.3. The four-holed sphere. When $\Sigma$ is a four-holed sphere, the relative character varieties admit a similar description. Present the fundamental group as

$$
\pi=\langle A, B, C, D \mid A B C D=1\rangle
$$

where the generators $A, B, C, D$ correspond to the components of $\partial \Sigma$. The elements

$$
X=A B, Y=B C, Z=C A
$$

correspond to simple closed curves on $\Sigma$. Denoting the trace functions $\operatorname{Hom}(\pi, G) / G \longrightarrow \mathbb{C}$ corresponding to elements $A, B, C, D, X, Y, Z \in \pi$ by lower-case, the relative character varieties are defined by:

$$
\begin{aligned}
x^{2}+y^{2}+z^{2}+x y z & =(a b+c d) x+(b c+a d) y \\
& +(a c+b d) z+\left(4-a^{2}-b^{2}-c^{2}-d^{2}-a b c d\right)
\end{aligned}
$$

with Poisson structure

$$
\begin{aligned}
\xi & =(a b+c d-2 x-y z) \partial_{y} \wedge \partial_{z} \\
& +(b c+d a-2 y-z x) \partial_{z} \wedge \partial_{x} \\
& +(c a+b d-2 z-x y) \partial_{x} \wedge \partial_{y} .
\end{aligned}
$$

1.5.4. The two-holed torus. Presenting the fundamental group of a twoholed torus as

$$
\pi=\langle A, B, X, Y \mid A X Y=Y X B\rangle,
$$

where $A, B \in \pi$ correspond to the two components of $\partial \Sigma$, the elements

$$
\begin{aligned}
Z & :=Y^{-1} X^{-1}, \\
U & :=A X Y=B Y X, \\
V & :=B Y, \\
W & :=A X
\end{aligned}
$$


are represented by simple closed curves. Using the same notation for trace coordinates as above, the relative character varieties are defined by the equations:

$$
\begin{aligned}
& a+b=x w+y v+u z-x y u \\
& \quad \begin{aligned}
a b= & x^{2}+y^{2}+z^{2}+u^{2}+v^{2}+w^{2} \\
& \quad+v w z-x y z-x u v-y u w-4
\end{aligned}
\end{aligned}
$$

and the Poisson structure is

$$
\begin{gathered}
(2 z-x y) \partial_{x} \wedge \partial_{y}+(2 x-y z) \partial_{y} \wedge \partial_{z}+(2 y-y x) \partial_{z} \wedge \partial_{x}+ \\
(2 u-v x) \partial_{v} \wedge \partial_{x}+(2 v-x u) \partial_{x} \wedge \partial_{u}+(2 x-u v) \partial_{u} \wedge \partial_{v}+ \\
(2 u-w y) \partial_{w} \wedge \partial_{y}+(2 w-y u) \partial_{y} \wedge \partial_{u}+(2 y-u w) \partial_{u} \wedge \partial_{w}+ \\
(2(x y-z)-v w) \partial_{v} \wedge \partial_{w}+(2(x u-v)-w z) \partial_{w} \wedge \partial_{z} \\
+(2(y u-w)-z v) \partial_{z} \wedge \partial_{v}
\end{gathered}
$$

These formulas are derived by applying the formulas for the Poisson bracket of trace functions developed in Goldman [38] in combination with the trace identities in $\operatorname{SL}(2, \mathbb{C}$ ) (see [46]).

\section{Compact Groups}

The simplest case occurs when $G=\mathrm{U}(1)$. Then

$$
\operatorname{Hom}(\pi, G) / G=\operatorname{Hom}(\pi, G) \cong \mathrm{U}(1)^{2 g} \cong H^{1}(\Sigma ; \mathbb{R} / \mathbb{Z})
$$

is a $2 g$-dimensional torus. If $M$ is a closed Riemann surface diffeomorphic to $\Sigma$, then $\operatorname{Hom}(\pi, G) / G$ identifies with the Jacobi variety of $M$, parametrizing topologically trivial holomorphic line bundles over $M$. Although the complex structures on $\operatorname{Hom}(\pi, G) / G$ vary with the complex structures on $\Sigma$, the symplectic structure is independent of $M$.

2.1. Ergodicity. The mapping class group action in this case factors through the symplectic representation

$$
\operatorname{Mod}_{\Sigma} \longrightarrow \operatorname{Sp}(2 g, \mathbb{Z})
$$

(since the representation variety is just the ordinary cohomology group with values in $\mathbb{R} / \mathbb{Z}$ ), which is easily seen to be ergodic. This generalizes to arbitrary compact groups:

Theorem 2.1. Let $G$ be a compact group. The Out $(\pi)$-action on $\operatorname{Hom}(\pi, G) / G$ is ergodic. 
When the simple factors of $G$ are locally isomorphic to $\mathrm{SU}(2)$ and $\Sigma$ is orientable, this was proved in Goldman [43]. For general $G$, this theorem is due to Pickrell-Xia [82] when $\Sigma$ is closed and orientable, and Pickrell-Xia [83] for compact orientable surfaces of positive genus.

The following conjecture generalizes the above ergodicity phenomenon:

Conjecture 2.2. Let $\Omega^{*}(\operatorname{Hom}(\pi, G) / G)$ be the de Rham algebra consisting of all measurable differential forms on $\operatorname{Hom}(\pi, G) / G$. Then the symplectic structures $\omega_{\mathbb{B}}$ generate the subalgebra of $\Omega^{*}(\operatorname{Hom}(\pi, G) / G)$ consisting of $\operatorname{Mod}_{\Sigma}$-invariant forms.

Since the $\mu$-measure of $\operatorname{Hom}(\pi, G) / G$ is finite, the representation of $\operatorname{Mod}_{\Sigma}$ on

$$
\left.\mathfrak{H}:=L^{2}(\operatorname{Hom}(\pi, G) / G, \mu)\right)
$$

is unitary. Andersen has informed me that he has proved vanishing of the first cohomology group $H^{1}\left(\operatorname{Mod}_{\Sigma}, \mathfrak{H}\right)$, and has raised the following conjecture generalizing Conjecture 2.2::

Conjecture 2.3. Suppose

$$
C^{\infty}(\operatorname{Hom}(\pi, G) / G) \stackrel{D}{\rightarrow} C^{\infty}(\operatorname{Hom}(\pi, G) / G)
$$

is a differential operator which commutes with the $\operatorname{Mod}_{\Sigma}$-action on $\operatorname{Hom}(\pi, G) / G$. Then $D$ is a scalar multiple of the identity operator.

2.2. The unitary representation. Ergodicity means that the only trivial subrepresentation of $\mathfrak{H}$ is the subspace $\mathbb{C}$ consisting of constants. Furthermore the action is weak mixing, by which we mean that $\mathbb{C}$ is the only finite-dimensional invariant subspace [43]. On the other hand the orthogonal complement $\mathfrak{H}_{0}$ to $\mathbb{C}$ in $\mathfrak{H}$ contains invariant subspaces. For example the closure of the span of trace functions of nonseparating simple closed curves on $\Sigma$ is an invariant subspace [48].

Problem 2.4. Decompose the representation on $\mathfrak{H}_{0}$ into irreducible representations of $\operatorname{Mod}_{\Sigma}$.

When $G=\mathrm{U}(1)$, and $\Sigma$ is the 2-torus, $\operatorname{Hom}(\pi, G) / G$ naturally identifies with $T^{2}$, by the functions $\alpha, \beta$ corresponding to a basis of $\pi_{1}(\Sigma)$. The functions

$$
\phi_{m, n}:=\alpha^{m} \beta^{n},
$$

forms a Hilbert basis of $\mathfrak{H}$, indexed by $(m, n) \in \mathbb{Z}^{2}$. The $\operatorname{Mod}_{\Sigma^{-}}$ representation on $\mathfrak{H}$ arises from the linear $\mathrm{GL}(2, \mathbb{Z})$-action on its basis $\mathbb{Z}^{2}$. The $\mathrm{GL}(2, \mathbb{Z})$-orbits on $\mathbb{Z}^{2}$ are indexed by integers $d \geq 0$. The orbit of $(d, 0)$ consists of all $(m, n) \in \mathbb{Z}^{2}$ with $\operatorname{gcd}(m, n)=d$. These are Hilbert bases for irreducible constituents $C_{d}$ of $\mathfrak{H}$. 
The irreducible constituents $C_{d}$ admit an alternate description, as follows. The $d$-fold covering homomorphism

$$
G \stackrel{\Phi_{d}}{\longrightarrow} G
$$

induces a covering space

$$
\operatorname{Hom}(\pi, G) / G \longrightarrow \operatorname{Hom}(\pi, G) / G \text {. }
$$

Let $L_{d}$ denote the closure of the image of the induced map $\mathfrak{H} \longrightarrow \mathfrak{H}$. Then

$$
L_{d}=\widehat{\bigoplus} C_{d^{\prime} \mid d}
$$

so $C_{d}$ consists of the orthocomplement in $L_{d}$ of the sum of all $L_{d^{\prime}}$ for $d^{\prime} \mid d$ but $d^{\prime} \neq d$.

Problem 2.5. Find a similar geometric interpretation for the irreducible constituents for compact nonabelian groups $G$.

2.3. Holomorphic objects. By Narasimhan-Seshadri [78], and Ramanathan [89], a marked conformal structure $(f, M)$ on $\Sigma$ interprets $\operatorname{Hom}(\pi, G) / G$ as a moduli space of holomorphic objects on $M$. To simplify the exposition we only consider the case $G=\mathrm{U}(n)$, for which $\operatorname{Hom}(\pi, G) / G$ identifies with the moduli space $\mathfrak{U}_{n}(M)$ of semistable holomorphic $\mathbb{C}^{n}$-bundles over $M$ of zero degree [78]. The union of all $\mathfrak{U}_{n}(M)$ over $\langle f, M\rangle$ in $\mathfrak{T}_{\Sigma}$ forms a holomorphic fiber bundle

$$
\mathfrak{U}_{n} \longrightarrow \mathfrak{T}_{\Sigma}
$$

with an action of $\operatorname{Mod}_{\Sigma}$. The quotient $\mathfrak{U}_{n} / \operatorname{Mod}_{\Sigma}$ fibers holomorphicly over $\mathfrak{M}_{\Sigma}$. The Narasimhan-Seshadri theorem gives a (non-holomorphic) map

$$
\mathfrak{U}_{n} \stackrel{\text { hol }}{\longrightarrow} \operatorname{Hom}(\pi, G) / G
$$

which on the fiber $\mathfrak{U}_{n}(M)$ is the bijection associating to an equivalence class of semistable bundles the equivalence class of the holonomy representation of the corresponding flat unitary structure. $\mathfrak{U}_{n} / \operatorname{Mod}_{\Sigma}$ inherits a foliation $\mathfrak{F}_{\mathfrak{U}}$ from the the foliation of $\mathfrak{U}_{n}$ by level sets of hol. The dynamics of this foliation are equivalent to the dynamics of the $\operatorname{Mod}_{\Sigma}$-action on $\operatorname{Hom}(\pi, G)$.

Go one step further and replace $\mathfrak{T}_{\Sigma}$ by its unit sphere bundle $U \mathfrak{T}_{\Sigma}$ and $\mathfrak{M}_{\Sigma}$ by its (orbifold) unit sphere bundle

$$
U \mathfrak{M}_{\Sigma}=\left(U \mathfrak{T}_{\Sigma}\right) / \operatorname{Mod}_{\Sigma}
$$

Pull back the fibration $\mathfrak{U}^{k}(\Sigma)$ to $U \mathfrak{M}_{\Sigma}$, to obtain a flat $\operatorname{Hom}(\pi, G) / G$ bundle $U \mathfrak{U}_{n}$ over $U \mathfrak{M}_{\Sigma}$, 
The Teichmüller geodesic flow is a vector field on $U \mathfrak{M}_{\Sigma}$ generating the geodesics for the Teichmüller metric on $\mathfrak{T}_{\Sigma}$. (Masur citeMasur) Its horizontal lift with respect to the flat connection is an vector field on the total space whose dynamics mirrors the dynamics of the $\operatorname{Mod}_{\Sigma^{-}}$ action on $\operatorname{Hom}(\pi, G) / G$.

As the $\operatorname{Mod}_{\Sigma}$-action on $\operatorname{Hom}(\pi, G) / G$ is weak-mixing, the unitary representation on $L^{2}(\operatorname{Hom}(\pi, G) / G, \mu)$ provides no nontrivial finitedimensional representations. Thus these representations markedly differ from the representations obtained by Hitchin [58] and Axelrod, Della-Pietra, and Witten [4] obtained from projectively flat connections on $\operatorname{Hom}(\pi, G) / G$. Recently Andersen [2] has proved that these finite-dimensional projective representations of $\operatorname{Mod}_{\Sigma}$ are asymptotically faithful.

2.4. Automorphisms of free groups. Analogous questions arise for the outer automorphism group of a free group $\pi$ of rank $r$. Let $G$ be a compact connected Lie group. Then Haar measure on $G$ defines an Out $(\pi)$-invariant probability measure on $\operatorname{Hom}(\pi, G)$.

Conjecture 2.6. If $r \geq 3$, the action of $\operatorname{Out}(\pi)$ on $\operatorname{Hom}(\pi, G)$ is ergodic.

Using calculations in [43], this conjecture has been proved [47] when all of the simple factors of $G$ are locally isomorphic to $\mathrm{SU}(2)$.

2.5. Topological dynamics. The topological theory is more subtle, since no longer may we ignore invariant subsets of measure zero. For example, if $F \subset G$ is a finite subgroup, then $\operatorname{Hom}(\pi, F)$ is finite and its image in $\operatorname{Hom}(\pi, G) / G$ is an invariant closed subset.

One might expect that if a representation $\rho \in \operatorname{Hom}(\pi, G)$ has dense image in $\operatorname{SU}(2)$, that the $\operatorname{Mod}_{\Sigma}$-orbit of $[\rho]$ is dense in $\operatorname{Hom}(\pi, G) / G$. This is true if $\Sigma$ is a one-holed torus (Previte-Xia [84]) and if the genus of $\Sigma$ is positive (Previte-Xia [85]). In genus 0 , representations $\rho$ exist with dense image but $\operatorname{Mod}_{\Sigma} \cdot[\rho]$ consists of only two points.

Similar examples exist when $\Sigma$ is a four-holed sphere. Benedetto and I showed [5], that when $-2<a, b, c, d<2$, the set of $\mathbb{R}$-points of the relative character variety has one compact component. This component is diffeomorphic to $S^{2}$. Depending on the boundary traces $(a, b, c, d)$, this component corresponds to either $\operatorname{SL}(2, \mathbb{R})$-representations or $\mathrm{SU}(2)$ representations. Previte and Xia [86] found representations $\rho$ in the components corresponding to $\mathrm{SL}(2, \mathbb{R})$-representations having dense image, but whose orbit $\left(\operatorname{Mod}_{\Sigma} \cdot[\rho]\right)$ has two points. On the other hand, in both cases, Previte and Xia [87] showed the action is minimal (every orbit is dense) for a dense set of boundary traces in $[-2,2]^{4}$. 
Problem 2.7. Determine necessary and sufficient conditions on a general representation $\rho$ for its orbit $\operatorname{Mod}_{\Sigma} \cdot[\rho]$ to be dense.

The case when $G=\mathrm{SU}(2)$ and $\Sigma$ an $n$-holed sphere for $n>4$ remains open.

2.6. Individual elements. For a closed surface of genus one, an individual element is ergodic on the SU(2)-character variety if and only if it is hyperbolic. In his doctoral thesis [13, 14], Brown used KAM-theory to show this no longer holds for actions on relative $\mathrm{SU}(2)$-character varieties over the one-holed torus. Combining Brown's examples with a branched-cover construction suggests:

Problem 2.8. Construct an example of a pseudo-Anosov mapping class for a closed surface which is not ergodic on the $\mathrm{SU}(2)$-character variety.

\section{Noncompact Groups and Uniformizations}

For noncompact $G$, one expects less chaotic dynamics. Trivial dynamics - in the form of proper Mod $_{\Sigma^{-}}$actions - occur for many invariant open subsets corresponding to locally homogeneous geometric structures, (in the sense of Ehresmann [27]) or uniformizations. Such structures are defined by local coordinate charts into a homogeneous space $G / H$ with coordinate changes which are restrictions of transformations from $G$. Such an atlas globalizes to a developing map, an immersion $\tilde{\Sigma} \longrightarrow G / H$ of the universal covering space $\tilde{\Sigma} \longrightarrow \Sigma$ which is equivariant with respect to a homomorphism $\pi \stackrel{\rho}{\rightarrow} G$.

To obtain a deformation space of such structures with an action of the mapping class group, one introduces markings for a fixed topological surface $\Sigma$, just as in the definition of Teichmüller space. The deformation space $\operatorname{Def}_{(G, G / H)}(\Sigma)$ consists of equivalence classes of marked $(G, G / H)$-structures with a holonomy map

$$
\operatorname{Def}_{(G, G / H)}(\Sigma) \stackrel{\text { hol }}{\longrightarrow} \operatorname{Hom}(\pi, G) / G
$$

which is $\operatorname{Mod}_{\Sigma}$-equivariant. The Ehresmann-Thurston theorem asserts that, with respect to an appropriate topology on $\operatorname{Def}_{(G, G / H)}(\Sigma)$, the mapping hol is a local homeomorphism. (This theorem is implicit in Ehresmann [28] and first explicitly stated by Thurston [98]. More detailed proofs were given by Lok [70], Canary-Epstein-Green [19], and Goldman [41]. Bergeron and Gelander [6] give a detailed modern proof with applications to discrete subgroups.)

If $G=\operatorname{PGL}(2, \mathbb{R})$ and $G / H=\mathrm{H}^{2}$ is the hyperbolic plane, then $\operatorname{Def}_{(G, G / H)}(\Sigma)=\mathfrak{T}_{\Sigma}$. 
Examples of uniformizations with proper $\operatorname{Mod}_{\Sigma}$-actions include:

- $G=\operatorname{PSL}(2, \mathbb{R})$ : The Teichmüller space $\mathfrak{T}_{\Sigma}$, regarded as the component of discrete embeddings in $\operatorname{Hom}(\pi, G) / G$;

- $G=\operatorname{PSL}(2, \mathbb{C})$ : Quasi-fuchsian space $\mathcal{Q}_{\Sigma}$ is an open subset of $\operatorname{Hom}(\pi, G) / G$ which is equivariantly biholomorphic to $\mathfrak{T}_{\Sigma} \times \overline{\mathfrak{T}_{\Sigma}}$;

- $G=\mathrm{SL}(3, \mathbb{R})$. The deformation space $\mathfrak{C}_{\Sigma}$ of convex $\mathbb{R P}^{2}$-structures is a connected component of $\operatorname{Hom}(\pi, G) / G$ (Choi-Goldman [20]) and the $\operatorname{Mod}_{\Sigma}$-action is proper. More generally if $G$ is a split $\mathbb{R}$-form of a semisimple group, Labourie [69] has shown that $\operatorname{Mod}_{\Sigma}$ acts properly on the contractible component of $\operatorname{Hom}(\pi, G) / G$ discovered by Hitchin [59].

3.1. Fricke-Teichmüller space. A Fuchsian representation of $\pi$ into $G=\operatorname{PSL}(2, \mathbb{R})$ is an isomorphism $\rho$ of $\pi=\pi_{1}(\Sigma)$ onto a discrete subgroup of $G$. Since $\pi$ is torsionfree and $\rho$ is injective, $\rho(\pi)$ is torsionfree. Hence it acts freely on $\mathrm{H}^{2}$ and the quotient $\mathrm{H}^{2} / \rho(\pi)$ is a complete hyperbolic surface. The representation $\rho$ defines a homotopy equivalence

$$
\Sigma \longrightarrow \mathrm{H}^{2} / \rho(\pi)
$$

which is homotopic to a homeomorphism. Thus $\rho$ is the holonomy homomorphism of a hyperbolic structure on $\Sigma$. The collection of $\operatorname{PGL}(2, \mathbb{R})$-conjugacy classes of such homomorphisms identifies (via the Uniformization Theorem) with the Teichmüller space $\mathfrak{T}_{\Sigma}$ of $\Sigma$. When $\partial \Sigma \neq \emptyset$, then the Fricke space is defined as the deformation space of complete hyperbolic structures on $\operatorname{lnt}(\Sigma)$ such that each end is either a cusp or a complete collar on a simple closed geodesic (a funnel). These representations map each component of $\partial \Sigma$ to either a parabolic or a hyperbolic element of $\operatorname{PSL}(2, \mathbb{R})$ respectively. For details on Fricke spaces see Bers-Gardiner [8].

The $\operatorname{Mod}_{\Sigma}$-action on $\mathfrak{T}_{\Sigma}$ is proper. This fact seems to have first been noted by Fricke [33] (see Bers-Gardiner [8] or Farb-Margalit [29]). It follows from two facts:

- $\operatorname{Mod}_{\Sigma}$ preserve a metric on $\mathfrak{T}_{\Sigma}$;

- The simple marked length spectrum

$$
\{\text { simple closed curves on } \Sigma\} / \operatorname{Diff}^{0}(\Sigma) \longrightarrow \mathbb{R}_{+}
$$

is a proper map.

See Abikoff [1], Bers-Gardiner [8], Farb-Margalit [29] or Harvey [56] for a proof. Another proof follows from Earle-Eels [24], and the closely 
related fact (proved by Palais and Ebin [25]) that the full diffeomorphism group of a compact smooth manifold acts properly on the space of Riemannian metrics. Compare [53].

3.2. Other components and the Euler class. Consider the case $G=\operatorname{PSL}(2, \mathbb{R})$. Then the components of $\operatorname{Hom}(\pi, G) / G$ are indexed by the Euler class

$$
\operatorname{Hom}(\pi, G) / G \stackrel{e}{\longrightarrow} H^{2}(\Sigma ; \mathbb{Z}) \cong \mathbb{Z}
$$

whose image equals

$$
\mathbb{Z} \cap[2-2 g-b, 2 g-2+b]
$$

where $\Sigma$ has genus $g$ and $b$ boundary components. Thus $\operatorname{Hom}(\pi, G) / G$ has $4 g+2 b-3$ connected components ([40] and Hitchin [57] when $b=0)$. The main result of [35] is that the two extreme components $e^{-1}( \pm(2-$ $2 g-b)$ ) consist of discrete embeddings. These two components differ by the choice of orientation, each one corresponding to $\mathfrak{T}_{\Sigma}$, upon which $\operatorname{Mod}_{\Sigma}$ acts properly. In contrast,

Conjecture 3.1. Suppose that $b=0$ ( $\Sigma$ is closed). For each integer $1 \leq k \leq 2 g+b-2$, the $\operatorname{Mod}_{\Sigma}$-action on the component $e^{-1}(2-2 g+b+k)$ of $\operatorname{Hom}(\pi, G)$ is ergodic.

When $b=0$, the component

$$
e^{-1}(3-2 g) \approx \Sigma \times \mathbb{R}^{6 g-8}
$$

represents a $6 g-6$-dimensional thickening of $\Sigma$, upon which $\operatorname{Mod}_{\Sigma}$ acts. However, Morita [81] showed that $\operatorname{Mod}_{\Sigma}$ cannot act smoothly on $\Sigma$ itself inducing the homomorphism $\operatorname{Diff}(\Sigma) \longrightarrow \operatorname{Mod}_{\Sigma}$. (Recently Markovic [80] has announced that if $\Sigma$ is a closed surface of genus $>5$, then $\operatorname{Mod}_{\Sigma}$ cannot even act on $\Sigma$ by homeomorphisms inducing $\operatorname{Homeo}(\Sigma) \longrightarrow \operatorname{Mod}_{\Sigma}$.)

Problem 3.2. Determine the smallest dimensional manifold homotopyequivalent to $\Sigma$ upon which $\operatorname{Mod}_{\Sigma}$ acts compatibly with the outer action of $\operatorname{Mod}_{\Sigma}$ on $\pi_{1}(\Sigma)$.

3.3. The one-holed torus. For surfaces with nonempty boundary, the dynamics appears more complicated. When $\Sigma$ is a one-holed torus $(g=b=1)$ and $G=\operatorname{PSL}(2, \mathbb{R})$ or $\operatorname{SU}(2)$, this was completely analyzed in [44].

As in $\S 1.5 .2$, the $\operatorname{SL}(2, \mathbb{C})$-character variety identifies with $\mathbb{C}^{3}$, where the three coordinates $(x, y, z)$ are traces of three generators of $\pi$ corresponding to the generators $X, Y, X Y$. In these coordinates, the trace 
of the element $K=X Y X^{-1} Y$ of $\pi$ corresponding to $\partial \Sigma$ equals

$$
\kappa(x, y, z):=x^{2}+y^{2}+z^{2}-x y z-2 .
$$

The relative $\operatorname{SL}(2, \mathbb{C})$-character variety of $\Sigma$ is then the family of level sets $\kappa^{-1}(t)$ of $\mathbb{C}^{3} \stackrel{\kappa}{\rightarrow} \mathbb{C}$.

The set $\kappa^{-1}(t) \cap \mathbb{R}^{3}$ of $\mathbb{R}$-points of $\kappa^{-1}(t)$, for boundary trace $t \in \mathbb{R}$, are of two types:

- The SU(2)-characters, with $x, y, z \in[-2,2]$ and $t<2$;

- The $\operatorname{SL}(2, \mathbb{R})$-characters, with either:

- at least one of $x, y, z$ lies in $(-\infty,-2] \cup[2, \infty)$, or

- each $x, y, z$ lies in $[-2,2]$ and $t \geq 2$.

If $|t|>2$, no $\mathrm{SU}(2)$-characters lie in $\kappa^{-1}(t) \cap \mathbb{R}^{3}$. If $t \neq 2$, these two subsets of $\kappa^{-1}(t) \cap \mathbb{R}^{3}$ are disjoint. If $t=2$, these two subsets intersect on the subset

$$
[-2,2]^{3} \cap \kappa^{-1}(2)
$$

corresponding to $\mathrm{SO}(2)$-representations. The space of $\mathrm{SO}(2)$-characters is 2-sphere with 4 branch points of cone angle $\pi$ (a tetrahedron with smoothed edges).

The $\operatorname{Mod}_{\Sigma}$-action determines a dynamical system on each level set. By Keen [65], the Fricke space of $\Sigma$ is the subset

$$
\left\{(x, y, z) \in \mathbb{R}^{3} \mid \kappa(x, y, z) \leq-2\right\}
$$

with a proper $\operatorname{Mod}_{\Sigma}$-action. Each level set $\mathbb{R}^{3} \cap \kappa^{-1}(t)$, for $t<-2$, is homeomorphic to a disjoint union of four discs; the four components are distinguished by different lifts of the representation from $\operatorname{PSL}(2, \mathbb{R})$ to $\operatorname{SL}(2, \mathbb{R})$.

The level set $\mathbb{R}^{3} \cap \kappa^{-1}(-2)$ has one notable feature. It has five components, four of which correspond to the Teichmüller space of $\Sigma$, and the other component $\{(0,0,0)\}$ consists of just the origin. The Teichmüller space (corresponding to the deformation space of complete finite area hyperbolic structures) corresponds to representations taking the boundary element of $\pi$ to a parabolic transformation of trace -2 . On the other hand, $\{(0,0,0)\}$ corresponds the quaternion representation in $\mathrm{SU}(2)$ :

$$
\begin{aligned}
& X \longmapsto\left[\begin{array}{cc}
i & 0 \\
0 & -i
\end{array}\right], \\
& Y \longmapsto\left[\begin{array}{cc}
0 & -1 \\
1 & 0
\end{array}\right]
\end{aligned}
$$

The peripheral element $K \in \pi$ maps to the nontrivial central element $-I \in \operatorname{SU}(2)$. 
Here we see - for the first time - the coexistence of two extremes of dynamical behavior:

- The proper action on the $\operatorname{SL}(2, \mathbb{R})$-characters;

- The entire mapping class group $\operatorname{Mod}_{\Sigma}$ fixes a point, in a sense, the "most chaotic" action.

This dichotomy persists for $-2<t<2$. The origin deforms to a compact component, consisting of characters of SU(2)-representations with an ergodic $\mathrm{Mod}_{\Sigma}$-action. Four contractible components, correspond to holonomy representations of hyperbolic structures on a torus with a cone point. The cone angle $\theta$ relates to the boundary trace by

$$
t=-2 \cos (\theta / 2) \text {. }
$$

The $\operatorname{Mod}_{\Sigma}$-action on these components is proper.

Although $\operatorname{Mod}_{\Sigma}$ acts properly, none of the corresponding representations are discrete embeddings. The key property seems to be that nonseparating simple loops are mapped to hyperbolic elements, so the simple marked length spectrum (3.1) is a proper map.

Problem 3.3. Find general conditions which ensure that (3.1) is proper.

The level set $\mathbb{R}^{3} \cap \kappa^{-1}(2)$ consists of characters of abelian representations, and $\operatorname{Mod}_{\Sigma}$ is ergodic on each of the four connected components of the smooth part of $\mathbb{R}^{3} \cap \kappa^{-1}(2)$. When $2<t \leq 18$, the $\operatorname{Mod}_{\Sigma}$-action on $\mathbb{R}^{3} \cap \kappa^{-1}(t)$ is ergodic.

For $t>18$, the level sets $\mathbb{R}^{3} \cap \kappa^{-1}(t)$ display both proper dynamics and chaotic dynamics. The region $(-\infty,-2]^{3}$ consists of characters of discrete embeddings $\rho$ where the quotient hyperbolic surface $\mathbf{H}^{2} / \rho(\pi)$ is homeomorphic to a three-holed sphere. Every homotopy equivalence $\Sigma \longrightarrow P$, where $P$ is a hyperbolic surface homeomorphic to a threeholed sphere, determines such a character. Furthermore these determine closed triangular regions which are freely permuted by $\operatorname{Mod}_{\Sigma}$. On the complement of these wandering domains the action is ergodic.

When $G=\operatorname{PGL}(2, \mathbb{R})$, the group of (possibly orientation-reversing) isometries of $\mathrm{H}^{2}$, a similar analysis was begun by Stantchev [94, 52]. One obtains similar dynamical systems, where $\operatorname{Mod}_{\Sigma}$ acts now on the space of representations into the group

$$
G_{ \pm}=\operatorname{SL}(2, \mathbb{C}) \cap(\mathrm{GL}(2, \mathbb{R}) \cup i \mathrm{GL}(2, \mathbb{R}))
$$

which doubly covers the two-component group PGL $(2, \mathbb{R})$. These $G_{ \pm^{-}}$ representations are again parametrized by traces. They comprise four components, one of which is the subset of $\mathbb{R}^{3}$ parametrizing $\operatorname{SL}(2, \mathbb{R})$ representations discussed above. The other three components are

$$
\mathbb{R} \times i \mathbb{R} \times i \mathbb{R}, \quad i \mathbb{R} \times \mathbb{R} \times i \mathbb{R}, \quad i \mathbb{R} \times i \mathbb{R} \times \mathbb{R}
$$


respectively. Consider $i \mathbb{R} \times \mathbb{R} \times i \mathbb{R}$. For $-14 \leq t<2$, the $\operatorname{Mod}_{\Sigma^{-}}$action is ergodic, but when $t<-14$, wandering domains appear. The wandering domains correspond to homotopy-equivalences $\Sigma \longrightarrow P$, where $P$ is a hyperbolic surface homeomorphic to a two-holed projective plane. The action is ergodic on the complement of the wandering domains.

Problem 3.4. Determine the ergodic behavior of the $\operatorname{Mod}_{\Sigma}$-action on the level sets

$$
(i \mathbb{R} \times \mathbb{R} \times i \mathbb{R}) \cap \kappa^{-1}(t)
$$

where $t>2$. The level sets for $t>6$ contains wandering domains corresponding to Fricke spaces of a one-holed Klein bottle.

3.4. Hyperbolic 3-manifolds. When $G=\operatorname{PSL}(2, \mathbb{C})$, the subset $\mathcal{Q}_{\Sigma}$ of $\operatorname{Hom}(\pi, G) / G$ corresponding to embeddings of $\pi$ onto quasi-Fuchsian subgroups of $G$ is open and $\operatorname{Mod}_{\Sigma^{-}}$-invariant. Furthermore the Bers isomorphism [7] provides a $\operatorname{Mod}_{\Sigma}$-invariant biholomorphism

$$
\mathcal{Q}_{\Sigma} \longrightarrow \mathfrak{T}_{\Sigma} \times \overline{\mathfrak{T}_{\Sigma}}
$$

Properness of the action of $\operatorname{Mod}_{\Sigma}$ on $\mathfrak{T}_{\Sigma}$ implies properness on $\mathcal{Q}_{\Sigma}$.

Points on the boundary of $\mathcal{Q}_{\Sigma}$ also correspond to discrete embeddings, but the action is much more complicated. Recently Souto and Storm [93] have proved that $\partial \mathcal{Q}_{\Sigma}$ contains a $\operatorname{Mod}_{\Sigma}$-invariant closed nowhere dense topologically perfect set upon which the action is topologically transitive. From this they deduce that every continuous $\operatorname{Mod}_{\Sigma^{-}}$ invariant function on $\partial \mathcal{Q}_{\Sigma}$ is constant.

While for representations into $G=\operatorname{PSL}(2, \mathbb{R})$, the $\operatorname{Mod}_{\Sigma}$-orbits of discrete embeddings are themselves discrete, the situation becomes considerably more complicated for larger $G$. For $G=\operatorname{PSL}(2, \mathbb{C})$, representations corresponding to the fiber of a hyperbolic mapping torus furnish points with infinite stabilizer. This is one of the easiest ways to see that $\operatorname{Mod}_{\Sigma}$ does not act properly on characters of discrete embeddings. Namely, if $M^{3}$ is a hyperbolic 3-manifold which admits a fibration $M^{3} \stackrel{f}{\rightarrow} S^{1}$, then the class of the restriction $\rho$ of the holonomy representation

$$
\pi_{1}\left(M^{3}\right) \longrightarrow \operatorname{PSL}(2, \mathbb{C})
$$

to the surface group

$$
\pi:=\pi_{1}\left(f^{-1}\left(s_{0}\right)\right) \cong \operatorname{Ker}\left(\pi_{1}(M) \stackrel{f_{*}}{\longrightarrow} \mathbb{Z}\right)
$$

is invariant under the monodromy automorphism $h \in \operatorname{Aut}(\pi)$ of $M^{3}$. That is, there exists $g \in \operatorname{PSL}(2, \mathbb{C})$ such that

$$
\rho(h(\gamma))=g \rho(\gamma) g^{-1}
$$


for all $\gamma \in \pi$. Furthermore $[\rho]$ is a smooth point of $\operatorname{Hom}(\pi, G) / G$. Kapovich [64] proved McMullen's conjecture [75] that the derivative of the mapping class $[h]$ at $[\rho]$ is hyperbolic, that is, no eigenvalue has norm 1. This contrasts the case of abelian representations, since homologically trivial pseudo-Anosov mapping classes act trivially on $\operatorname{Hom}(\pi, G)$.

Thus $\operatorname{Mod}_{\Sigma}$ does not act properly on the set of characters of discrete embeddings. Let $[\rho]$ (as above) be the character of a discrete embedding of $\pi$ as the fiber of a hyperbolic mapping torus. The stabilizer of $[\rho]$ contains the infinite cyclic group generated by the mapping class corresponding to $[h]$. (In fact $\langle[h]\rangle$ has finite index in the stabilizer of $[\rho]$.) Since stabilizers of proper actions of discrete groups are finite, $\operatorname{Mod}_{\Sigma}$ does not act properly.

The Souto-Storm theorem shows that this chaotic dynamical behavior pervades the entire boundary of quasi-Fuchsian space $\mathcal{Q}_{\Sigma}$.

In another direction, using ideas generalizing those of Bowditch [10], Tan, Wong and Zhang [97] have shown that the action of $\operatorname{Mod}_{\Sigma}$ on the representations satisfying the analogue of Bowditch's Q-conditions is proper. This also generalizes the properness of the action on the space of quasi-Fuchsian representations.

At present little is known about the dynamics of $\operatorname{Mod}_{\Sigma}$ acting on the $\operatorname{SL}(2, \mathbb{C})$-character variety. Conversations with Dumas led to the following problem:

Problem 3.5. Find a point $\rho \in \operatorname{Hom}(\pi, \operatorname{SL}(2, \mathbb{C}))$ such that the closure of its orbit $\overline{\operatorname{Mod}_{\Sigma} \cdot[\rho]}$ meets both the image of the unitary characters $\operatorname{Hom}(\pi, \mathrm{SU}(2))$ and the closure $\overline{\mathcal{Q}_{\Sigma}}$ of the quasi-Fuchsian characters.

3.4.1. Homological actions. The action of $\operatorname{Mod}_{\Sigma}$ on the homology of $\operatorname{Hom}(\pi, G) / G$ furnishes another source of possibly interesting linear representations of $\operatorname{Mod}_{\Sigma}$. With Neumann [51], we proved that for the relative $\operatorname{SL}(2, \mathbb{C})$-character varieties of the one-holed torus and fourholed sphere, the action of $\operatorname{Mod}_{\Sigma}$ factors through a finite group.

Atiyah-Bott [3] use infinite-dimensional Morse theory to analyze the algebraic topology of $\operatorname{Hom}(\pi, G) / G$, when $G$ is compact. For the nonsingular components their techniques imply that the $\operatorname{Mod}_{\Sigma^{-}}$-action on the rational cohomology of $\operatorname{Hom}(\pi, G) / G$ factors through the symplectic representation of $\operatorname{Mod}_{\Sigma}$ on $H^{*}(\Sigma)$. In particular Biswas [9] proved that the Torelli group acts trivially on nonsingular components. In contrast, Cappell-Lee-Miller [17, 18] proved the surprising result that that the Torelli group acts nontrivially on the homology of the SU(2)character variety when $\Sigma$ is closed. 


\subsection{Convex Projective Structures and Hitchin representations.} When $G=\operatorname{SL}(3, \mathbb{R})$, the mapping class group $\operatorname{Mod}_{\Sigma}$ acts properly on the component $\mathfrak{C}_{\Sigma}$ of $\operatorname{Hom}(\pi, G) / G$ corresponding to convex $\mathbb{R P}^{2}$ structures (Goldman [42], Choi-Goldman [20, 21]). This component is homeomorphic to a cell of dimension $-8 \chi(\Sigma)$, and, for a marked Riemann surface $M$ homeomorphic to $\Sigma$, admits the natural structure of a holomorphic vector bundle over $\mathfrak{T}_{\Sigma}$. The work of Labourie [68] and Loftin $[71,72,73]$ gives a more intrinsic holomorphic structure on $\mathfrak{C}_{\Sigma}$.

The existence of this contractible component is a special case of a general phenomenon discovered by Hitchin [59]. Hitchin finds, for any split real form of a semisimple group $G$, a contractible component in $\operatorname{Hom}(\pi, G) / G$. For $G=\operatorname{SL}(n, \mathbb{R})$ this component is characterized as the component containing the composition of discrete embeddings

$$
\pi \longrightarrow \mathrm{SL}(2, \mathbb{R})
$$

with the irreducible representation

$$
\mathrm{SL}(2, \mathbb{R}) \longrightarrow \mathrm{SL}(n, \mathbb{R}) .
$$

Recently, Labourie has found a dynamical description [69] of representations in Hitchin's component, and has proved they are discrete embeddings. Furthermore he has shown that $\operatorname{Mod}_{\Sigma}$ acts properly on this component. (These closely relate to the higher Teichmüller spaces of Fock-Goncharov [30, 31].)

When $G$ is the automorphism group of a Hermitian symmetric space of noncompact type, Bradlow, Garcia-Prada, and Gothen have found other components of $\operatorname{Hom}(\pi, G) / G$, for which the Toledo invariant, is maximal $[11,12]$. Their techniques involve Morse theory along the lines of Hitchin [57]. Recently, Burger, Iozzi, and Wienhard have shown [15] that the representations of maximal Toledo invariant consist of discrete embeddings. Using results from [16], Wienhard has informed me that $\operatorname{Mod}_{\Sigma}$ acts properly on these components,

3.6. The energy of harmonic maps. An interesting invariant of surface group representations arises from the theory of twisted harmonic maps of Riemann surfaces, developed in detail in collaboration with Wentworth [53]. Namely to each reductive representation $\pi \stackrel{\rho}{\rightarrow} G$, one associates an energy function

$$
\mathfrak{T}_{\Sigma} \stackrel{E_{\rho}}{\longrightarrow} \mathbb{R}
$$

whose qualitative properties reflect the $\operatorname{Mod}_{\Sigma}$-action. Assuming that the Zariski closure of $\rho(\pi)$ in $G$ is reductive, for every marked Riemann 
surface $\Sigma \longrightarrow M$, there is a $\rho$-equivariant harmonic map

$$
\tilde{M} \longrightarrow G / K
$$

where $K \subset G$ is a maximal compact subgroup (Corlette [22], Donaldson [23], Labourie [67], and Jost-Yau [63], following earlier work by Eels-Sampson [26]). Its energy density determines an exterior 2-form on $\Sigma$, whose integral is defined as $E_{\rho}(\langle f, M\rangle)$.

When $\rho(\pi)$ lies in a compact subgroup of $G$, then the twisted harmonic maps are constant, and the energy function is constantly zero. At the other extreme is the following result, proved in [53]:

Theorem 3.6. Suppose that $\rho$ is an embedding of $\pi$ onto a convex cocompact discrete subgroup of $G$. Then the energy function $E_{\rho}$ is a proper function on $\mathfrak{T}_{\Sigma}$.

Here a discrete subgroup $\Gamma \subset G$ is convex cocompact if there exists a geodesically convex subset $N \subset G / K$ such that $\Gamma \backslash N$ is compact. For $\operatorname{PSL}(2, \mathbb{C})$, these are just the quasi-Fuchsian representations. This result was first proved by Tromba [99] for Fuchsian representations in $\operatorname{PSL}(2, \mathbb{R})$, and the ideas go back to Sacks-Uhlenbeck [90] and SchoenYau [91].

It is easy to prove (see [53]) that if $\Omega \subset \operatorname{Hom}(\pi, G) / G$ is $\operatorname{aod}_{\Sigma^{-}}$ invariant open set for which each function $E_{\rho}$ is proper, for $[\rho] \in \Omega$, then the action of $\operatorname{Mod}_{\Sigma}$ on $\Omega$ is proper. This gives a general analytic condition implying properness.

Unfortunately, convex cocompactness is extremely restrictive; Kleiner and Leeb have proved [66] that in rank $>1$ such groups are never Zariski dense. However, we know many examples (the deformation space $\mathfrak{C}_{\Sigma}$ of convex $\mathbb{R P}^{2}$-structures, the Hitchin representations by Labourie [69], other components of maximal representations [11, 12, 16]) where we expect the $\operatorname{Mod}_{\Sigma}$-action to be proper. The only use of geodesic convexity in the above result is that the images of harmonic maps are constrained to lie in the set $N$.

Problem 3.7. Find a substitute for convex cocompactness in higher rank which includes the above examples of proper $\operatorname{Mod}_{\Sigma}$-actions, and for which $E_{\rho}$ is proper.

The work of Bonahon-Thurston on geometric tameness, and its recent extensions, implies that the energy function of a discrete embed$\operatorname{ding} \pi \longrightarrow \operatorname{PSL}(2, \mathbb{C})$ is proper if and only if it is quasi-Fuchsian [53].

3.7. Singular uniformizations and complex projective structures. When $G=\operatorname{PSL}(2, \mathbb{R})$, the other components of $\operatorname{Hom}(\pi, G)$ may 
be studied in terms of hyperbolic structures with singularities as follows. Instead of requiring all of the coordinate charts to be local homeomorphisms, one allows charts which at isolated points look like the map

$$
\begin{aligned}
& \mathbb{C} \longrightarrow \mathbb{C} \\
& z \mapsto z^{k}
\end{aligned}
$$

that is, the geometric structure has an isolated singularity of cone angle $\theta=2 k \pi$. Such a singular hyperbolic structure may be alternatively described as a singular Riemannian metric $g$ whose curvature equals -1 plus Dirac distributions weighted by $2 \pi-\theta_{i}$ at each singular point $p_{i}$ of cone angle $\theta_{i}$. The structure is nonsingular on the complement $\Sigma-\left\{p_{1}, \ldots, p_{k}\right\}$, and that hyperbolic structure has holonomy representation

$$
\pi_{1}\left(\Sigma-\left\{p_{1}, \ldots, p_{k}\right\}\right) \stackrel{\hat{\rho}}{\rightarrow} \operatorname{PSL}(2, \mathbb{R})
$$

such that the holonomy of a loop $\gamma_{i}$ encircling $p_{i}$ is elliptic with rotation angle $\theta_{i}$.

In particular if each $\theta_{i} \in 2 \pi \mathbb{Z}$, then $\hat{\rho}\left(\gamma_{i}\right)=1$. The representation $\hat{\rho}$ extends to a representation $\rho$ of $\pi_{1}(\Sigma)$ :



Applying Gauss-Bonnet to $g$ implies that $\rho$ has Euler class

$$
e(\rho)=\chi(M)+\frac{1}{2 \pi} \sum_{i=1}^{k}\left(\theta_{i}-2 \pi\right) .
$$

It is convenient to assume that each $\theta_{i}=4 \pi$ and the points $p_{i}$ are not necessarily distinct - a cone point of cone angle $4 \pi$ with multiplicity $m$ is then a cone point with cone angle $2(m+1) \pi$. The uniformization theorem of McOwen [77], Troyanov [100], and Hitchin [57] asserts: given a Riemann surface $M \approx \Sigma$, there exists a unique singular hyperbolic structure in the conformal class of $M$ with cone angle $\theta_{i}$ at $x_{i}$ for $i=1, \ldots, k$ as long as

$$
\chi(\Sigma)+\frac{1}{2 \pi} \sum_{i=1}^{k}\left(\theta_{i}-2 \pi\right)<0 .
$$


(Hitchin only considers the case when $\theta_{i}$ are multiples of $2 \pi$, while McOwen and Troyanov deal with arbitrary positive angles.) The resulting uniformization map assigns to the collection of points $\left\{p_{1}, \ldots, p_{k}\right\}$ (where $0 \leq k \leq|\chi(\Sigma)|$ ) the singular hyperbolic structure with cone angles $4 \pi$ (counted with multiplicity) at the $p_{i}$. The equivalence class of the holonomy representation in the component

$$
e^{-1}(\chi(\Sigma)+k) \subset \operatorname{Hom}(\pi, G) / G
$$

defines a map from the symmetric product $\operatorname{Sym}^{k}(M)$ to $e^{-1}(\chi(M)+k)$. The following result follows from Hitchin [35]:

Theorem 3.8. Let $M$ be a closed Riemann surface. The above map

$$
\operatorname{Sym}^{k}(M) \longrightarrow e^{-1}(\chi(M)+k)
$$

is a homotopy equivalence.

The union of the symmetric powers $\operatorname{Sym}^{k}(M)$, one for each marked Riemann surface $M$, over $\langle f, M\rangle \in \mathfrak{T}_{\Sigma}$, can be given the structure of a holomorphic fiber bundle $\mathfrak{S}^{k}(\Sigma)$ over $\mathfrak{T}_{\Sigma}$, to which the action of $\operatorname{Mod}_{\Sigma}$ on $\mathfrak{T}_{\Sigma}$ lifts. The above maps define a homotopy equivalence

$$
\mathfrak{S}^{k}(\Sigma) \stackrel{\mathbb{U}}{\longrightarrow} e^{-1}(\chi(\Sigma)+k) .
$$

which is evidently $\operatorname{Mod}_{\Sigma}$-equivariant. However, since $\operatorname{Mod}_{\Sigma}$ acts properly on $\mathfrak{T}_{\Sigma}$, it also acts properly on the $(6 g-6+2 k)$-dimensional space $\mathfrak{S}^{k}(\Sigma)$. The quotient $\mathfrak{S}^{k}(\Sigma) / \operatorname{Mod}_{\Sigma}$ is the total space of an (orbifold) $\operatorname{Sym}^{k}(\Sigma)$-bundle over the Riemann moduli space

$$
\mathfrak{M}_{\Sigma}:=\mathfrak{T}_{\Sigma} / \operatorname{Mod}_{\Sigma}
$$

The fibers of $\mathbb{U}$ define a (non-holomorphic) foliation of $\mathfrak{S}^{k}(\Sigma) / \operatorname{Mod}_{\Sigma}$, a flat $\mathrm{Sym}^{k}(\Sigma)$-bundle, whose dynamics mirrors the dynamics of the $\operatorname{Mod}_{\Sigma}$-action on the component $e^{-1}(\chi(\Sigma)+k)$.

In general, $\mathbb{U}$ is not onto: if $\Sigma \stackrel{f}{\rightarrow} \Sigma^{\prime}$ is a degree one map to a closed surface $\Sigma^{\prime}$ of smaller genus, and $\rho^{\prime}$ is a Fuchsian representation of $\pi_{1}\left(\Sigma^{\prime}\right)$, then $\rho:=\rho^{\prime} \circ f_{*}$ lies outside Image $(\mathbb{U})$. The following conjecture arose in discussions with Neumann:

Conjecture 3.9. If $k=1$, then $\mathbb{U}$ is onto. In general a $\operatorname{PSL}(2, \mathbb{R})$ representation with dense image lies in Image(U).

3.8. Complex projective structures. A similar construction occurs with the deformation space $\mathbb{C P}^{1}(\Sigma)$ of marked $\mathbb{C P}^{1}$-structures on $\Sigma$. A $\mathbb{C P}^{1}$-manifold is a manifold with a coordinate atlas modeled on $\mathbb{C P}^{1}$, with coordinate changes in $G=\operatorname{PSL}(2, \mathbb{C})$. The space $\mathbb{C P}^{1}(\Sigma)$ consists 
of equivalence classes of marked $\mathbb{C P}^{1}$-structures, that is, homeomorphisms $\Sigma \longrightarrow N$ where $N$ is a $\mathbb{C P}^{1}$-manifold. Since $\operatorname{PSL}(2, \mathbb{C})$ acts holomorphicly, the $\mathbb{C P}^{1}$-atlas is a holomorphic atlas and $N$ has a underlying Riemann surface $M$. The resulting $\operatorname{Mod}_{\Sigma^{-}}$equivariant map

$$
\mathbb{C P}^{1}(\Sigma) \longrightarrow \mathfrak{T}_{\Sigma}
$$

is a holomorphic affine bundle, whose underlying vector bundle is the holomorphic cotangent bundle of $\mathfrak{T}_{\Sigma}$. In particular $\operatorname{Mod}_{\Sigma}$ acts properly on $\mathbb{C P}^{1}(\Sigma)$ with quotient a holomorphic affine bundle over $\mathfrak{M}_{\Sigma}$.

The map which associates to a marked $\mathbb{C P}^{1}$-structure on $\Sigma$ its holonomy representation is a local biholomorphism

$$
\mathbb{C P}^{1}(\Sigma) \stackrel{\text { hol }}{\longrightarrow} \operatorname{Hom}(\pi, G) / G
$$

which is known to be very complicated. Gallo-Kapovich-Marden [34] have shown that its image consists of all equivalence classes of representations $\rho$ for which:

- $\rho$ lifts to a representation $\pi \longrightarrow \mathrm{SL}(2, \mathbb{C})$;

- The image $\rho(\pi)$ is not precompact;

- The image $\rho(\pi)$ is not solvable.

The latter two conditions are equivalent to $\rho(\pi)$ not leaving invariant a finite subset of $\mathrm{H}^{3} \cup \partial \mathrm{H}^{3}$. (The cardinality of this finite subset can be taken to be either 1 or 2.)

The holonomy map hol is $\operatorname{Mod}_{\Sigma}$-equivariant. The action of $\operatorname{Mod}_{\Sigma}$ on $\mathbb{C P}^{1}(\Sigma)$ is proper, since it covers the action of $\operatorname{Mod}_{\Sigma}$ on $\mathfrak{T}_{\Sigma}$. The quotient $\mathbb{C P}^{1}(\Sigma) / \operatorname{Mod}_{\Sigma}$ affinely fibers over $\mathfrak{M}_{\Sigma}$. As before hol defines a foliation of $\mathbb{C P}^{1}(\Sigma) / \operatorname{Mod}_{\Sigma}$ orbit equivalent to the $\operatorname{Mod}_{\Sigma}$-action on $\operatorname{Hom}(\pi, G) / G$. Thus hol may be regarded as a resolution of the $\operatorname{Mod}_{\Sigma^{-}}$ action.

As a simple example, the $\operatorname{Mod}_{\Sigma}$-action is proper on the quasi-Fuchsian subset $\mathcal{Q}_{\Sigma} \subset \operatorname{Hom}(\pi, G) / G$. As noted above, it is a maximal open set upon which $\operatorname{Mod}_{\Sigma}$ acts properly. Its restriction

$$
\operatorname{hol}^{-1}\left(\mathcal{Q}_{\Sigma}\right) \longrightarrow \mathcal{Q}_{\Sigma}
$$

is a covering space $([39])$. However, the bumping phenomenon discovered by McMullen [76] implies that hol is not a covering space on any open neighborhood strictly containing $\mathcal{Q}_{\Sigma}$.

\section{REFERENCES}

[1] Abikoff, W., "The Real Analytic Theory of Teichmüller Space," Lecture Notes in Mathematics 820, Springer-Verlag, Berlin, 1980.

[2] Andersen, J., Asymptotic faithfulness of the quantum SU(n)-representations of the mapping class groups, Ann. Math. (to appear), math.QA/0204084. 
[3] Atiyah, M., and Bott, R., The Yang-Mills equations over Riemann surfaces, Phil. Trans. R. Soc. Lond. A 308

[4] Axelrod, S., Della Pietra, S., and Witten, E., Geometric quantization of ChernSimons gauge theory, J. Diff. Geom. 33 (1991), 787-902. .

[5] Benedetto, R. and Goldman, W., The Topology of the Relative Character Varieties of a Quadruply-Punctured, Sphere, Experimental Mathematics (1999) 8:1, $85-104$.

[6] Bergeron, N. and Gelander, T., A note on local rigidity, Geom. Ded. 107 (2004), 111-131.

[7] Bers, L., Simultaneous uniformization, Bull. Amer. Math. Soc. 66, (1960), 94-97.

[8] and F. Gardiner, Fricke spaces, Adv. Math. 62 (1986), 249-284.

[9] Biswas, I., On the mapping class group action on the cohomology of the representation space of a surface, Proc. Amer. Math. Soc. 124 (1996), no. 6, 1959-1965.

[10] Bowditch, B. H., Markoff triples and quasi-Fuchsian groups, Proc. London Math. Soc. (3) 77 (1998), no. 3, 697-736.

[11] Bradlow, S., Garcia-Prada, O., and Gothen, P., Surface group representations in PU $(p, q)$ and Higgs bundles, J. Diff. Geo. 64 (2003), no. 1, 111-170.

[12] _ Surface group representations, Higgs bundles, and holomorphic triples, math. AG/0206012.

[13] Brown, R., "Mapping class actions on the SU(2)-representation varieties of compact surfaces," Doctoral dissertation, University of Maryland (1996).

[14] _ Anosov mapping class actions on the SU(2)-representation variety of a punctured torus, Ergod. Th. \& Dynam. Sys. 18 (1998), 539-544.

[15] Burger, M., Iozzi, A., and Wienhard, A., Surface group representations with maximal Toledo invariant, C. R. Math. Acad. Sci. Paris, Ser. I 336 (2003), 387-390.

[16] Burger, M., Labourie, F., Iozzi, A. and Weinhard, A., Maximal representations of surface group: Symplectic Anosov structures, DG/0506079.

[17] Cappell, S., Lee, R., and Miller, E., The Torelli group action on respresentation spaces, in "Geometry and Topology: Aarhus 1998", 47-70, Contemp. Math. 258, Amer. Math. Soc., Providence, RI, 2000.

[18] _ The action of the Torelli group on the homology of representation spaces is nontrivial, Topology 39 (2000), no. 4, 851-871.

[19] Canary, R., Epstein, D. and Green, P. Notes on notes of Thurston, "Analytical and Geometrical Aspects of Hyperbolic Space (Coventry/Durham 1984)," London Math. Soc. Lecture Note Series 111, Cambridge Univ. Press (1987), $3-92$.

[20] Choi, S., and Goldman, W., Convex real projective structures on closed surfaces are closed, Proc. A.M.S. 118 No.2 (1993), 657-661.

[21] _ The Classification of Real Projective Structures on compact surfaces, Bull. A.M.S. (New Series) 34, No. 2, (1997), 161-170.

[22] Corlette, K., Flat bundles with canonical metrics, J. Diff. Geom. 28 (1988), $361-382$.

[23] Donaldson, S., Twisted harmonic maps and the self-duality equations, Proc. London Math. Soc. (3)55 (1987), 127-131. 
[24] Earle, C.J. and Eels, J., A fibre bundle description of Teichmüller theory, J. Diff. Geom. 3 (1969), 19-43.

[25] D. Ebin, The manifold of Riemannian metrics, in "Global Analysis" (Proc. Sympos. Pure Math. Vol. XV, Berkeley, Calif.), 11-40, (1968), Amer. Math. Soc., Providence, R. I.

[26] Eels, J. and Sampson, J., Harmonic mappings of Riemannian manifolds, Amer. J. Math. 86 (1964), 109-160.

[27] Ehresmann, C., Sur les espaces localement homogenes, L'ens. Math. 35 (1936), $317-333$

[28]_Les connexions infinitesimales dans un fibré differentiable, Extrait du "Colloque de Topologie," Bruxelles (1950), CBRM.

[29] Farb, B., and Margalit, D., "A primer on mapping class groups," (in preparation).

[30] Fock, V., and Goncharov,A., Moduli spaces of local systems and higher Teichmüller theory, math.AG/0311149 (submitted).

[31] _ Moduli spaces of convex projective structures on surfaces, math.DG/0405348 (submitted).

[32] Fock, V., and Rosly, A., Poisson structure on moduli of flat connections on Riemann surfaces and r-matrix,. Am. Math. Soc. Transl. 191 (1999) 67-86. math.QA/9802054.

[33] Fricke, R., and Klein, F., "Vorlesungen uber der Automorphen Funktionen", vol. I (1897)

[34] Gallo, D., Kapovich, M. and Marden, A., The monodromy groups of Schwarzian equations on closed Riemann surfaces, Ann. Math. 151 (2000), 625-704.

[35] Goldman, W. , "Discontinuous groups and the Euler class," Doctoral dissertation, University of California, Berkeley (1980).

[36] _. The symplectic nature of fundamental groups of surfaces, Adv. Math. Vol. 54 (1984), pp. 200-225.

[37] _., Representations of fundamental groups of surfaces, in "Geometry and Topology, Proceedings, University of Maryland 1983-1984", J. Alexander and J. Harer (eds.), Lecture Notes in Mathematics 1167 95-117, Springer-Verlag (1985).

[38] _ Invariant functions on Lie groups and Hamiltonian flows of surface group representations, Inv. Math. 85 (1986), 1-40.

[39] _ Projective structures with Fuchsian holonomy, J. Diff. Geom. 25 (1987), 297-326.

[40] _ Topological components of spaces of representations, Inv. Math. 93 (3), (1988), 557-607.

[41] _ Geometric structures and varieties of representations, in "The Geometry of Group Representations," Contemp. Math. 74 , Amer. Math. Soc. (1988), 169-198.

[42] Convex real projective structures on compact surfaces, J. Diff. Geo. 31 (1990), 791-845.

[43] _ Ergodic theory on moduli spaces, Ann. Math. 146 (1997), 1-33

[44] Action of the modular group on real SL(2)-characters of a one-holed torus, Geometry and Topology 7 (2003), 443-486. mathDG/0305096. 
[45] _ The complex symplectic geometry of $S L(2, C)$-characters over surfaces, in "Proceedings of International Conference on Algebraic Groups and Arithmetic," December 17-22, 2001, TIFR, Mumbai. math.DG/0304307.

[46] _ An exposition of results of Fricke, math. GM/0402103 (submitted).

[47] An ergodic action of the outer automorphism group of a free group, math.DG/0506401 (submitted)

[48] _ The mapping class group acts reducibly on SU(2)-character varieties, preprint (2005), to appear in "Knots and Primes," Proceedings of 2003 JAMI Workshop, Johns Hopkins University.

[49] _ and Millson,J., Deformations of flat bundles over Kähler manifolds, in "Geometry and Topology, Manifolds, Varieties, and Knots," C. McCrory and T.Shifrin (eds.), Lecture Notes in Pure and Applied Mathematics 105 (1987), 129-145 Marcel Dekker Inc., New York Basel.

[50] $\quad$, The deformation theory of representations of fundamental groups of Kähler manifolds, Publ. Math. I. H. E. S. 67 (1988),43-96.

[51] Goldman, W. and Neumann, W., Homological action of the modular group on some cubic moduli spaces, Math. Research Letters (to appear).math.GT/0402039

[52] Goldman, W. and Stantchev, G., Dynamics of the Automorphism Group of the GL(2,R)-Characters of a Once-punctured Torus, math.DG/0309072 (submitted).

[53] Goldman, W. and Wentworth, R., Energy of Twisted Harmonic Maps of Riemann Surfaces, math.DG/0506212 (submitted).

[54] Goldman, W. and Xia, E., Rank one Higgs bundles on Riemann surfaces and representations of fundamental groups, math.DG/0402429 Memoirs A.M.S. (to appear).

[55] Guruprasad, K., Huebschmann, J., Jeffrey, L., Weinstein, A., Group systems, groupoids, and moduli spaces of parabolic bundles, Duke Math. J. 89 No. 2, (1997), 377-4121.

[56] Harvey, W. J., Spaces of Discrete Groups, in "Discrete Groups and Automorphic Functions," Academic Press (1977), 295-347.

[57] Hitchin, N., The self-duality equations on Riemann surfaces, Proc. Lond. Math. Soc. 55 (1987), 59-126.

[58] , Flat connections and geometric quantization, Commun. Math. Phys. 131 (1990), 347-380.

[59] _ Lie groups and Teichmüller space, Topology 31 (3), (1992), 449-473.

[60] Huebschmann, J., Symplectic and Poisson structures of certain moduli spaces, Duke Math J. 80 (1995) 737-756.

[61] Jeffrey,L. and Weitsman, J., Bohr-Sommerfeld orbits and the Verlinde dimension formula, Commun. Math. Phys. 150 (1992) 593-630

[62] _ Toric structures on the moduli space of flat connections on a Riemann surface: volumes and the moment map, Advances in Mathematics 109, 151168 (1994).

[63] Jost, J. and Yau, S.T., Harmonic maps and group representations, Differential Geometry, H.B. Lawson and K. Tenenblat, eds., Longman, pp. 241-259.

[64] Kapovich, M., On the dynamics of pseudo-Anosov homeomorphisms on representation varieties of surface groups, Ann Acad. Sci. Fenn. Math. 23 (1998) no.1, 83-100. 
[65] Keen, L., On Fricke Moduli, in "Advances in the Theory of Riemann Surfaces," Ann. Math. Studies 66 (1971), 205-224.

[66] Kleiner, B. and Leeb, B., Rigidity of invariant convex sets in symmetric spaces, math.DG/0412123.

[67] Labourie, F., Existence d'applications harmoniques tordues à valeurs dans les variétés à courbure négative, Proc. Amer. Math. Soc. 111 (1991), 877-882.

$[68] \_, \mathbb{R P}^{2}$-structures et differentielles cubiques holomorphes, Proc. of the GARC Conference in Differential Geometry, Seoul National University (1997).

[69] _ Anosov flows, surface group representations and curves in projective space, math.DG/0401230 (submitted).

[70] Lok, W., "Deformations of locally homogeneous spaces and Kleinian groups," Doctoral dissertation, Columbia University (1984).

[71] Loftin, J., Applications of Affine Differential Geometry to $\mathbb{R P}^{2}$ Surfaces, Doctoral dissertation, Harvard University (1999).

[72] _ Affine spheres and convex $\mathbf{R} P^{n}$-manifolds, Amer. J. Math. 123 (2001), 255-274.

[73] _ The compactification of the moduli space of convex $\mathbb{R P}^{2}$-surfaces I, (submitted).

[74] Masur, H., Interval exchange transformations and measured foliations, Ann. Math. 115 (1982), no. 1, 169-200.

[75] McMullen, C., "Renormalization and 3-manifolds which fiber over the circle," Ann. Math. Studies 142, Princeton University Press (1996).

[76] _ Complex earthquakes and Teichmüller theory, J. Amer. Math. Soc. 11 (1998), 283-320.

[77] McOwen, R., Prescribed curvature and singularities of conformal metrics on Riemann surfaces, J. Math. Anal. Appl. 177 (1993), no. 1, 287-298.

[78] Narasimhan, M. S. and Seshadri, C. S., Stable and unitary vector bundles over compact Riemann surfaces, Ann. Math. 82 (1965), 540-567.

[79] Lerman, E., and Sjamaar, R. Stratified spaces and reduction, Ann. Math. (2) 134 (1991), no. 2, 375-422.

[80] Markovic, V., Realization of the Mapping Class Group by Homeomorphisms, preprint available from http://www.maths.warwick.ac.uk/ markovic/.

[81] Morita,S., Characteristic classes of surface bundles, Bull. Amer. Math. Soc. (N.S) 11 (1984), no. 2, 386-388.

[82] Pickrell, D. and Xia, E., Ergodicity of Mapping Class Group Actions on Representation Varieties, I. Closed Surfaces, Comment. Math. Helv. 77 (2001), $339-362$.

[83] _ Ergodicity of Mapping Class Group Actions on Representation Varieties, II. Surfaces with Boundary, Transformation Groups 8 (2003), no. 4, 397-402.

[84] Previte, J., and Xia, E., Topological dynamics on moduli spaces I, Pac. J. Math. 193 (200), no. 2, 397-417.

[85] _ Topological dynamics on moduli spaces II, Trans. Amer. Math. Soc. 354 (2002), no. 6, 2475-2494.

[86] __ Exceptional discrete mapping class group orbits in moduli spaces, Forum Math. 15 (2003), no. 6, 949-954.

[87] _ Dynamics of the Mapping Class Group on the Moduli of a Punctured Sphere with Rational Holonomy, Geom. Ded. 112 (2005) (to appear). 
[88] Raghnunathan, M. S.,. "Discrete Subgroups of Lie Groups" Springer-Verlag Ergebnisse (1972).

[89] Ramanathan, A., Moduli for principal bundles over algebraic curves, Proc. Indian Acad. Sci. Math. Sci. 106 (1996) I no.3, 301-326, II no.4, 421-449

[90] J. Sacks and K. Uhlenbeck, Minimal immersions of closed Riemann surfaces, Trans. Amer. Math. Soc. 271 (1982), 639-652.

[91]_ and S.T.Yau, Existence of incompressible minimal surfaces and the topology of three-dimensional manifolds with non-negative scalar curvature, Ann. Math. 110 (1979), 127-142.

[92] Simpson, C. T., Nonabelian Hodge theory, Proc. I.C.M., Kyoto 1990, SpringerVerlag (1991), 198-230.

[93] Souto, J., and Storm, P., Dynamics of the mapping class group action on the variety of PSL(2,C) characters. math.GT/0504474 (submitted).

[94] Stantchev, G., "Action of the modular group on GL(2,R)-characters on a oncepunctured torus," Doctoral dissertation, University of Maryland (2003).

[95] Sullivan, D., Quasiconformal homeomorphisms and dynamics. II. Structural stability implies hyperbolicity for Kleinian groups, Acta Math. 155 (1985), no. 3-4, 243-260.

[96] Tan, S. P., Branched $\mathbb{C P}^{1}$-structures with prescribed real holonomy, Math. Ann. bf 300 (1994), 649-667.

[97] _ and Wong, Y. L. and Zhang, Y., Generalized Markoff maps and McShane's identity, math.GT/0502464 (submitted).

[98] Thurston, W., "The geometry and topology of 3-manifolds," Princeton University lecture notes (1979) (unpublished).

[99] Tromba, A., "Teichmüller Theory in Riemannian Geometry," ETHZ Lectures in Mathematics, Birkhäuser (1992).

[100] Troyanov, M., Prescribing curvature on compact surfaces with conical singularities, Trans. Amer. Math. Soc. 324 (1991), no. 2, 793-821.

[101] Vogt, H., Sur les invariants fondamentaux des equations différentielles linéaires du second ordre, Ann. Sci. L'École Normale Supérieure, $3^{\text {eme }}$ Série, Tome VI, (1889) Supplement $1-70$.

[102] Weil, A. On discrete subgroups of Lie groups II, Ann. Math. 75 (1962), 578602 .

Department of Mathematics, University of Maryland, College Park, MD 20742

E-mail address: wmg@math.umd.edu 